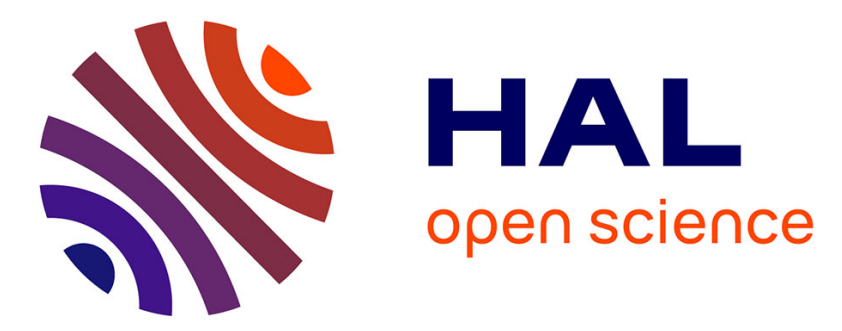

\title{
Intermittent statistics of the 0 -mode pressure fluctuations in the near field of Mach 0.9 circular jets at low and high Reynolds numbers

\author{
R Camussi, Christophe Bogey
}

\section{- To cite this version:}

R Camussi, Christophe Bogey. Intermittent statistics of the 0-mode pressure fluctuations in the near field of Mach 0.9 circular jets at low and high Reynolds numbers. Theoretical and Computational Fluid Dynamics, 2021, 35, pp.229 - 247. 10.1007/s00162-020-00553-9 . hal-03210344

\section{HAL Id: hal-03210344 \\ https://hal.science/hal-03210344}

Submitted on 28 Apr 2021

HAL is a multi-disciplinary open access archive for the deposit and dissemination of scientific research documents, whether they are published or not. The documents may come from teaching and research institutions in France or abroad, or from public or private research centers.
L'archive ouverte pluridisciplinaire HAL, est destinée au dépôt et à la diffusion de documents scientifiques de niveau recherche, publiés ou non, émanant des établissements d'enseignement et de recherche français ou étrangers, des laboratoires publics ou privés. 


\title{
Intermittent statistics of the $\mathbf{0}$-mode pressure fluctuations in the near field of Mach 0.9 circular jets at low and high Reynolds numbers
}

R. Camussi ${ }^{\mathrm{a} 1}$, C. Bogey

a Università degli Studi Roma TRE, Dipartimento di Ingegneria, Via della Vasca Navale 79, 00146

Rome, Italy

${ }^{b}$ Univ Lyon, Ecole Centrale de Lyon, INSA Lyon, Universitè Claude Bernard Lyon I, CNRS, Laboratoire de Mècanique des Fluides et d'Acoustique, UMR 5509, F-69134, Ecully, France

\begin{abstract}
The present paper reports an investigation of the statistical properties of pressure fluctuations in the near field of subsonic compressible jets. The data-base analysed has been obtained numerically by DNS and LES of two single-stream circular jets, having diameter-based Reynolds numbers of 3,125 and 100,000 and Mach number 0.9, respectively initially laminar and highly disturbed. Pressure fluctuations are extracted from several virtual probes positioned in the near field of the jets and covering a region from 0 to 20 diameters in the axial direction and from 0.5 to 3 diameters in the radial. An azimuthal decomposition of the pressure fluctuations is performed, and the statistical analysis is applied to the axisymmetric 0-mode component and compared to the results obtained from the full original signals. The intermittent behaviour is investigated by the estimation of standard statistical indicators, such as Probability Distribution Functions and Flatness Factor, as well as through conditional statistics based on the application of the wavelet transform. It is shown that downstream of the potential core, intermittency estimated through the traditional indicators is relevant even at the lowest Re for the full signals whereas it is apparently not significant for the 0mode component. The wavelet analysis provides an estimation of intermittency scale-by-scale and allows for the calculation of a frequency dependent FF. This approach reveals that the 0-mode component has a relevant degree of intermittency around the frequencies associated to the KelvinHelmholtz instability. The statistics of the intermittent events, in terms of their temporal appearance and energy content, are shown to be weakly sensitive to the jet Reynolds number and the universal behaviour can be reproduced by simple stochastic models.
\end{abstract}

\section{Keywords}

Jet noise; Intermittency; Stochastic modelling

\section{Declarations}

Not applicable

\section{Introduction}

\footnotetext{
${ }^{1}$ Corresponding author roberto.camussi@uniroma3.it
} 
The first seminal studies performed to investigate subsonic jet noise go back to more than sixty years [1-2], however, despite the large body of literature devoted to the subject since then, several questions still remain unanswered and deserve further investigations. It is known that jet-noise properties at small and large observation angles to the flow are very different (see also [3] and [4]). At small observer angle, the low-frequency acoustic component dominates, and the noise emission can be attributed to large turbulence structures that are spatially coherent in the axial direction. These structures, named wavepackets, can be viewed as the growth and decay of linear instability waves (see [5] and [6]) originated by the Kelvin-Helmholtz instability of the shear layer and convected downstream by the mean flow (see the review provided by Jordan \& Colonius [7], and the reference therein).

Seminal papers by Crow [8] and Crighton \& Huerre [9] demonstrated the sound radiation superdirectivity towards low axial angles (see also [10]) and its relationship with the axisymmetric structures of the jets. This evidence supported the idea of using linear models to predict the radiated noise, but this approach led to satisfactory results only in supersonic conditions ([11] and [12]). The main feature that was missing in the subsonic case was the random nature of the hydrodynamic events responsible for the noise generation. Indeed, at high Reynolds numbers (Re), the dynamics of those structures is non-deterministic since turbulence significantly affects their generation, evolution and topology. Recent papers highlighted the relevant contribution of turbulence-induced intermittency and non-linearities to the generation of noise [13-15]. Actually, the role of intermittency in the noise generation mechanism has been recognized since the seminal experiment undertaken in [16] and by successive studies [17-22] that confirmed the existence and dominant role played by intermittent noise producing events.

In the framework of jet noise models based on the wavepacket concepts, it is argued that those large scale structures emerge from the stochastic turbulent flow (see the seminal paper by Crighton and Gaster [23] and [24]). Indeed, the paper by Cavalieri et al. [25], for the first time demonstrated that the simplified wavepacket model is able to predict correct noise levels only if the so-called jittering mechanism is included in the source model (see also the review in [26]). Recent papers have shown that a manifestation of jitter in the frequency domain is given by the decay of the two-point coherence in the velocity and near pressure fields [27-31]. Indeed, the stochastic, intermittent nature of the flow causes a desynchronisation of flow fluctuations separated in space and thus directly affects the coherence decay. Two-point coherence values extracted directly from a subsonic jet have been used to feed a wavepacket model for sound radiation at low frequencies and polar angles, providing a good agreement with acoustic data [13]. The advantage of this approach is its direct correlation with the acoustic efficiency of sound sources according to the concept of acoustic matching related to the content of energy falling within the radiating region of the spectrum [27, 29].

The complex nature of the jet noise sources can be taken into account by considering its multipole nature. A quadrupole type of noise source combined with a proper correlation model, has been shown to be an efficient approach to provide predictions in good agreement with experimental data (see e.g. [32] and [33]). With respect to the wavepacket modeling, in this approach the time scales the modelled sources correspond to those of small-scale eddies instead of those of instability waves. Also this model is capable of tackling the stochastic properties of the noise source and confirmed that the bursting of turbulent small-scale eddies contributes a significant part to the generated noise. 
The awareness that the stochastic nature of jet noise needs to be better understood, motivated a series of studies devoted to the investigation and modelling of the intermittent statistics by selecting the events directly in the physical space using appropriate indicators applied to near-field pressure data (see e.g. [34-35]). Recently, Camussi et al. [36-37] used wavelet transform to select events related to wavepackets and proposed stochastics models to reproduce their relevant statistics. This approach provides a more direct measure of the degree of intermittency contained in the pressure field and allows for the extraction of local features that are partially lost through the Fourier-based approach. Indeed, the main target was to retrieve the properties of the stochastic field that can be used for the noise source description and modelling. The present paper moves within this path and concurs to clarify the stochastic behavior underlying the dynamics of the noise producing events. The present investigation is based on the analysis of a numerical database reproducing two jets at Mach number (Ma) 0.9 and at significantly different Reynolds numbers (Re) [38]. As will be better clarified below, the relevant number of "virtual probes" located in the jet flow (more than 24,000) renders this numerical database incomparable with respect to any possible experimental test. Furthermore, the numerical approach allows for two significantly different $\operatorname{Re}(3,125$ and 100,000$)$ to be investigated using the same nozzle geometry and at the same Ma. The signals are decomposed azimuthally and the analysis is carried out on the 0-mode component as well. The purpose is to correlate more directly the achieved results with the noise generation mechanisms.

The main scope of the paper is to evaluate the degree of intermittency of the pressure field in the vicinity of the jet with particular focus on the lowest Re where the flow appears more coherent and quasi-laminar. Similarly to the approach developed in [36] and [37], wavelet analysis is applied to extract energetic intermittent events and to retrieve their statistical properties. With respect to those previous studies, a wavelet-based indicator more directly related to intermittency is introduced and used to select events and compute conditional statistics. The achieved results are useful to explore the statistics of the near-field pressure and, as a major novelty, to clarify the dynamics underlying the 0 -mode pressure fluctuations and to highlight the effect of the Reynolds number on the intermittent statistics. The procedure adopted for the statistical investigation is described in detail in Sec. 2. This section provides also a brief description of the numerical simulation originating the data analyzed therein. Results are presented in Sec. 3 whereas Sec. 4 reports the main conclusions. 


\section{Numerical set-up and post-processing procedures}

The analyzed numerical database was built for the two isothermal round jets at a Mach number $\mathrm{M}$ $=0.9$ and Reynolds numbers $R e=U D / v$ of 100,000 and 3,125, where $U, D$ and $v$ are the flow velocity at the jet exit, the jet diameter and the kinematic molecular viscosity, considered in a recent work [38]. Both jets originate from a pipe nozzle of length $D$ into a medium at ambient temperature and pressure $293 \mathrm{~K}$ and $10 \mathrm{e} 5 \mathrm{~Pa}$. At the nozzle exit, the mean velocity profiles correspond to Blasius laminar boundary-layer profiles of thickness 0.075D for the high Re case and 0.21D for the low Re case. Peak axial turbulence intensities of $9.14 \%$ and of $0.99 \%$ are prescribed by forcing the boundary layer in the pipe [25]. Therefore, the two jets are initially highly disturbed and laminar, respectively. The potential core length is equal to 7.3D for both jets.

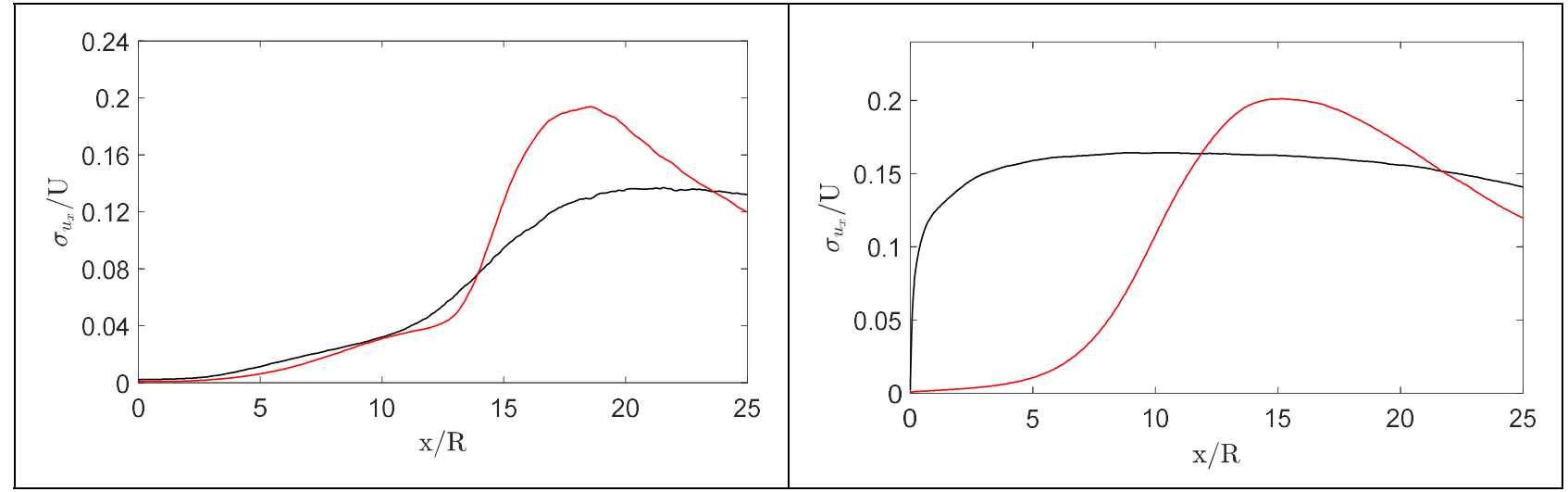

Figure 1: Normalized axial velocity $r m s$ as a function of $x / R$ (where $R$ is the jet radius). The black curves correspond to the high Re case whereas red curves to low Re. The plot on the left corresponds to a radial location $r=0$, the plot on the right to $r=R$.

In order to better clarify the transitional nature of the jet flow at the two Re analyzed, we report in Figure 1 the profiles of $r m s$ axial velocities computed on the jet axis $(r=0)$ and in the shear layer $(r=R$ where $R=D / 2$ ) for the two jets considered. The profiles obtained at $r=R$ show in particular that for the high $R e$ case the jet shear layers are transitional between $x=0$ and $x \approx 3 R$ and turbulent farther downstream. On the other hand, for the low Re case, they are laminar between $x=0$ and $x \approx 6 R$, then transitional down to $x=18 \mathrm{R}$ and turbulent farther downstream. In the latter case, there is a strong peak of turbulence levels due to the laminar-turbulent transition, occurring approximately at the location of the pairings of the first vortical structures formed after the shear layer rolling up.

The simulation of the high Re jet is a well-resolved LES. As detailed in a previous grid-sensitivity study [39], the mesh grid contains 2085, 512 and 1024 points in the axial (x), radial ( $r$ ) and azimuthal ( $\phi)$ directions, and extends, excluding the sponge zones, up to 20D axially and 7.5D radially. The maximal mesh spacing in the jet near field is equal to $0.037 \mathrm{D}$, leading to a Strouhal number of $\mathrm{St}_{\mathrm{D}}=$ $\mathrm{fD} / \mathrm{U}=5.9$ for an acoustic wave discretized by five points per wavelength, where $f$ is the frequency. The simulation of the low Re jet is a direct numerical simulation. In this case, the mesh grid is made of 2458,427 and 512 points, and extends up to $37.5 \mathrm{D}$ and $12.5 \mathrm{D}$ in the axial and radial directions. The maximal mesh spacing is doubled compared to the high Re case. 


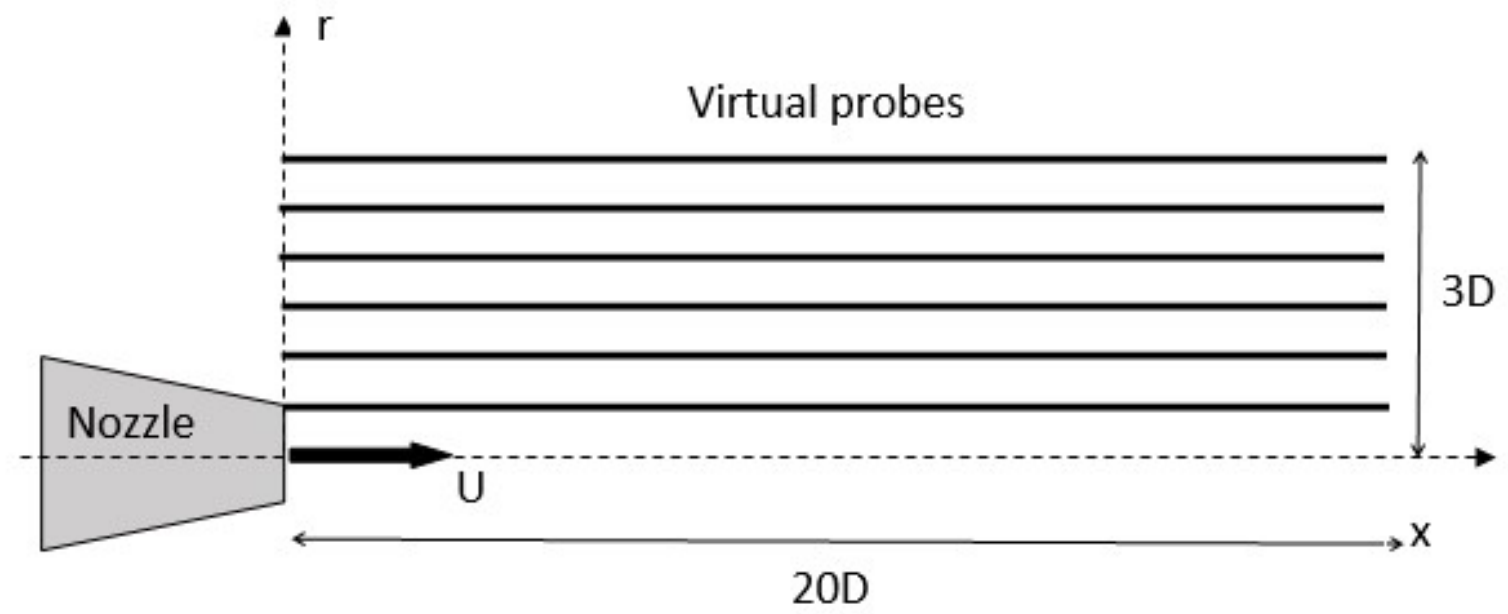

Figure 2: A simplified scheme representing the flow domain and the virtual probes location. $U$ denotes the mean velocity at the jet exit; $x$ is the axial coordinate and $r$ the radial coordinate. 1024 probes are displaced along each bold line parallel to the $x$-axis and regularly separated by $r / D=0.5$ in the radial direction.

The simulation times after the transient periods are equal to $1,000 \mathrm{D} / \mathrm{U}$ for the high $\mathrm{Re}$ jet and to $1,500 \mathrm{D} / \mathrm{U}$ for the low Re jet. Density, velocities and pressure obtained for the azimuthal angles of 0 , $45^{\circ}, 90^{\circ}, 135^{\circ}, 180^{\circ}, 225^{\circ}, 270^{\circ}, 315^{\circ}$ for the low Re jet and of $0,90^{\circ}, 180^{\circ}$ and $270^{\circ}$ for the high $\operatorname{Re}$ jet are recorded at a sampling frequency allowing spectra to be computed up to $S t_{D}=6.4$.

More information about the numerical setup and the jet initial conditions can be found in [38-41].

The original pressure signals consist of time series $p(x, r, \varphi, t)$ that are represented in terms of their azimuthal components through the azimuthal decomposition of the Fourier modes. This approach has been extensively used in the past to extract relevant features connected with the jet noise generation. The Azimuthal Decomposition provided the identification of the relevant acoustic mode dominating the pressure and the velocity field in the mixing region in low and high $\mathrm{M}$ number subsonic jets (see e.g. 31 and ref. from 41 to 44). In the present analysis, the azimuthal mode spectra were determined using the classical theory of Michalke and Fuchs [42] which uses the orthogonality property of a Fourier cosine series. In the compact exponential form, the decomposition is provided by the following simple expression:

$$
p(x, r, \varphi, t)=\sum_{m=-\infty}^{\infty} \hat{p}_{m}(x, r, t) e^{i m \varphi}
$$

where $\hat{p}_{m}$ is the complex-valued pressure Fourier transform of mode order $\mathrm{m}$. The Fourier coefficients are stored for the first nine azimuthal modes even though the analysis presented therein is limited to the axisymmetric case $m=0$, that is known to dominate the sound field for low polar angles [9-10]. This property has been checked to characterize also the sound directivity of the two jets presently analysed (results are not reported for the sake of brevity).

To the purposes of the present analysis, a set of virtual probes are extracted with the scope of investigating in great details the statistics of the near field pressure fluctuations. The region analyzed spans from $x / D=0$ to 20 and from $r / D=0.5$ to 3 . The probes are distributed along 6 straight lines 
aligned with the jet axis. Along each line, 1024 probes are displaced with a constant separation and pressure time series are extracted from each one. The scheme is repeated for each azimuthal angle to the scope of increasing the number of realizations and improve the statistical convergence, being the random pressure field statistically axisymmetric. The total number of virtual probes is of about 24,000 and a pressure time signal is extracted from each one. A simplified sketch of the probe displacement is reported in Figure 1.

The numerical data are analyzed using standard procedures, including Fourier transform and Probability Density Functions (PDFS). The latter quantities are always referred to normalized variables obtained by subtracting to the random variable its mean value and dividing it by the standard deviation. In order to evaluate the degree of intermittency, the Flatness Factor (FF) of the pressure time series $p(t)$ is computed according to the following standard definition:

$$
F F=\frac{\left\langle p^{\prime 4}\right\rangle}{\left\langle p^{\prime 2}\right\rangle^{2}}
$$

where $\mathrm{p}^{\prime}$ denotes the fluctuating (zero mean) component of the pressure signal and the symbol $\langle\ldots\rangle$ denotes averaging.

It should be noted that, due to the intrinsic limitation of the numerical approach, the maximum number of samples per each virtual probe is limited (of the order of $7 \times 10^{4}$ ) and thus, statistical moments of order higher than the fourth could not be computed accurately.

The conditional statistics is retrieved through the analysis of the pointwise pressure time series in the wavelet domain. The wavelet transform is obtained by the convolution of the pressure signal $\mathrm{p}(t)$ with the dilated (by the factor $s$ ) and translated (by the factor $\tau$ ) complex conjugate counterpart of a so-called mother wavelet $\psi(t)$, according to the following formalism:

$$
w(s, t)=\frac{1}{\sqrt{s}} \int_{-\infty}^{+\infty} \Psi^{*}\left(\frac{\tau-t}{s}\right) p(\tau) d \tau
$$

where the factor $1 / \sqrt{s}$ ensures that the scaled mother wavelet has the same energy for all scales $s$ [46], the parameter $s$ being proportional to the Fourier frequency (see [47] for the details). In the present approach, the wavelet transform uses the Morlet kernel that is well suited for ensuring the energy conservation [47]. The wavelet analysis is carried out in the Matlab framework using the "Time-Frequency Toolbox" developed by Auger et al. [48] and available freely.

A key quantity that will be analyzed in the following is the local (in terms of time and frequency) energy of the given signal. This energy map is retrieved by the wavelet scalogram, obtained by the time-frequency distribution of the square of the wavelet coefficients: $w^{2}(s, t)$.

As pointed out in [36], the scalogram may be interpreted as a projection in the time domain of the Fourier modes and the standard Fourier spectrum can be retrieved by its integration in time (see [46] and [49]).

In a statistical sense, intermittency is intended as a sequence of quiescent phases interrupted by active events inducing a non-homogeneous distribution of energy in time. According to [46] and [50] a wavelet based quantity able to identify intermittent events is the so-called Local Intermittency 
Measure (LIM), a normalized version of the wavelet scalogram. The formal definition of LIM is the following:

$$
\operatorname{LIM}(s, t)=\frac{w^{2}(s, t)}{\left\langle w^{2}(s, t)\right\rangle_{t}}
$$

where the symbol $\langle\ldots\rangle_{t}$ denotes averaging in time. The condition $L I M>1$ identifies regions where the energy is larger than the mean and thus permits the localization of relevant, in terms of energy content, events in time and scale. To the purpose of identifying events related to intermittency, Meneveau [51] suggested that a more suited indicator is the LIM squared, LIM² (hereafter denoted as LIM2)

$$
\operatorname{LIM} 2(s, t)=\frac{w^{4}(s, t)}{\left\langle w^{2}(s, t)\right\rangle_{t}{ }^{2}}
$$

Indeed, LIM2 can be interpreted as a time-scale (or time-frequency) dependent measure of the FF of the input signal.

The LIM2 can be averaged in time in order to retrieve a depiction of the FF as a function of frequency. This quantity is defined as WFF:

$$
W F F(s)=\langle\operatorname{LIM} 2(s, t)\rangle_{t}=\frac{\left\langle w^{4}(s, t)\right\rangle_{t}}{\left\langle w^{2}(s, t)\right\rangle_{t}{ }^{2}}
$$

it provides an efficient way to discover which is the most intermittent frequency contained by a signal.

To the extent of the conditional statistics, LIM2 represents a convenient tool to extract those features contributing to the deviation from Gaussianity. Indeed, LIM2 is equal to 3 if the probability distribution is Gaussian, consequently the condition LIM2 $>3$ identifies only those events contributing to the departure from a Gaussian distribution of the wavelet coefficients. The events tracking identification technique adopted therein is based on this concept and is formalized as follows:

$$
\{\tilde{s}, \tilde{t}\}: \operatorname{LIM} 2(s, t)=\frac{w^{4}(s, t)}{\left\langle w^{2}(s, t)\right\rangle_{t}{ }^{2}}>3
$$

$\{\tilde{s}, \tilde{t}\}$ denotes the set of scales (or frequency) and temporal locations of the identified events. Three indicators are extracted from the selected events:

(i) The delay between consecutive events. This quantity is computed from the set $\tilde{t}$ and it is denoted as:

$$
\Delta t(i)=\tilde{t}(i+1)-\tilde{t}(i)
$$

(ii) The mean frequency of the events. It is obtained by averaging $\tilde{s}$ and is made nondimensional using $U$ and D. It is denoted as MIF (Mean Intermittent Frequency)

(iii) The energy of the events, determined by the square of the corresponding (at the same scale and time of the selected events) wavelet coefficients, i.e. $w^{2}(\tilde{s}, \tilde{t})$ 
The statistical properties of those indicators are analyzed in detail in the following section along with the global statistics. As pointed out above, an azimuthal decomposition of the pressure signals is performed and the statistical analysis is extended to the pressure signals reconstructed from the mode zero (denoted as 0-mode pressure). According to [36] and [37], stochastic models of the conditional statistics are proposed in order to provide reliable predictions of the effects of the governing parameters, namely $x / D, r / D$ and Re.

\section{Results}

\subsection{Overall statistics}

The statistical properties of the pressure fluctuations are influenced significantly by the presence of the jet flow and by the vortical structures generated in the shear layer and convected downstream by the mean stream. In the region very close to the jet, pressure is dominated by hydrodynamic fluctuations (usually called pseudo-sound, see e.g. [52]) induced by the vorticity field whereas for increasing distances, the hydrodynamic contribution decays very fast according to an exponential decay law [52-53] and only the acoustic component survives in the far field.

As shown e.g. by Arndt et al. [43], the impact of the hydrodynamic pressure fluctuations in the near field of the jet is revealed by a low frequency bump in the pressure power spectral density (PSD). The frequency corresponding to the PSD maxima is associated with the Kelvin-Helmholtz mode and will be denoted as $\mathrm{f}_{\mathrm{KH}}$ [36-37]. It corresponds to a Strouhal number (St $\mathrm{KH}_{\mathrm{H}}$ ) that ranges from 0.1 to 0.5 (see also [4] and [7]). Examples are reported in Figure 3 for the two Reynolds numbers analyzed therein. The presence of a bump is evident and the $\mathrm{f}_{\mathrm{KH}}$ (and thus $S \mathrm{t}_{\mathrm{KH}}$ ) can be definitely determined from the corresponding frequency. Similar results were reported in [38] for larger r/D.

Due to the intrinsic limitations of the Fourier decomposition, the spectral analysis does not clarify the dynamics of the pressure fluctuations. The hydrodynamic pressure fluctuations, being induced by the flow structures, are expected to be governed by a non-Gaussian statistic and to be characterized by the presence of intermittent pressure drops induced by the random vorticity. This is a well-known behavior (see e.g. [54-56] ) and can be qualitatively inferred from Figure 4 where a segment of two pressure time series taken at the two Re considered and close to the end of the potential core are reported. In both cases, the presence of high-intensity pressure drops is evident. Even though qualitatively, the two pressure time series seem to exhibit a very similar statistic. 

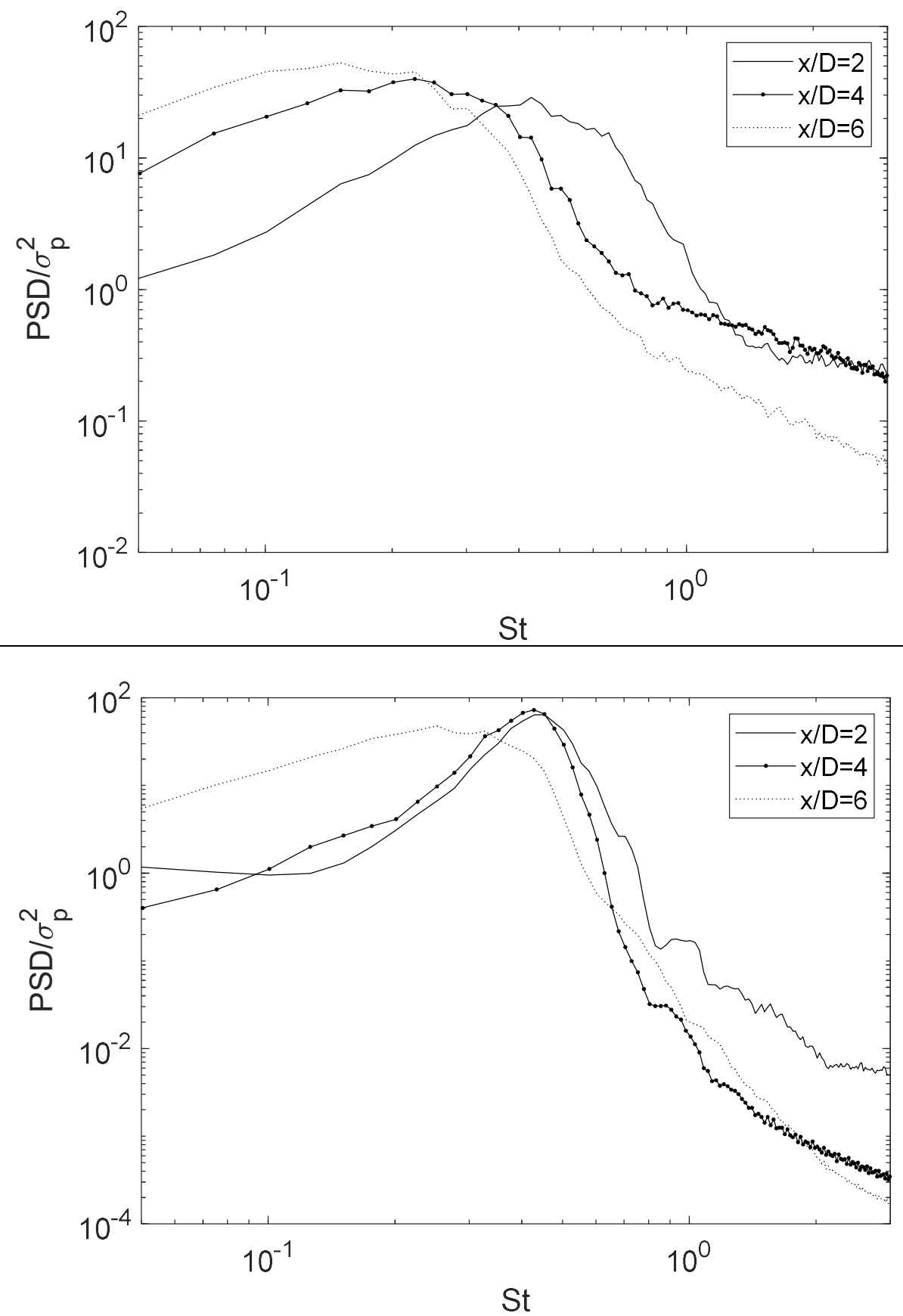

Figure 3: Pressure PSD normalized by the signals variance taken at high (top) and low (bottom) Re for $r / D=1$. The presence of the hydrodynamic bump associated to the Kelvin-Helmholtz mode can be clearly inferred.

This outcome can be quantitatively substantiated by the analysis of the Probability Distribution Functions (PDFs) of the pressure fluctuations. The presence of intermittent pressure drops on the overall statistics of the pressure fluctuations is expected to be revealed by an increase of the negative tails of the PDFs as well as by an increase of their flatness factor (FF) caused by the relevant weight of those rare, but very intense, events. Both effects are indeed confirmed by the analysis that follows. The pressure PDFs computed at a reference distance from the jet nozzle $(x / D=6)$ and 
for two distinct radial positions $(r / D=1$ and 3 ) are presented in semi-log scale in Figures 5 for the low and high Re. The selected location is close to the end of the potential core in both cases and the effect of intermittency is highlighted by the comparison against a reference Gaussian curve. It is clearly observed that for small r/D, the PDF is non-Gaussian and it is characterized by a predominant negative tail representing the statistical signature of the pressure drops. On the other hand, the pressure fluctuations at large r/D have Gaussian statistics as an effect of the disappearance of the hydrodynamic effects.

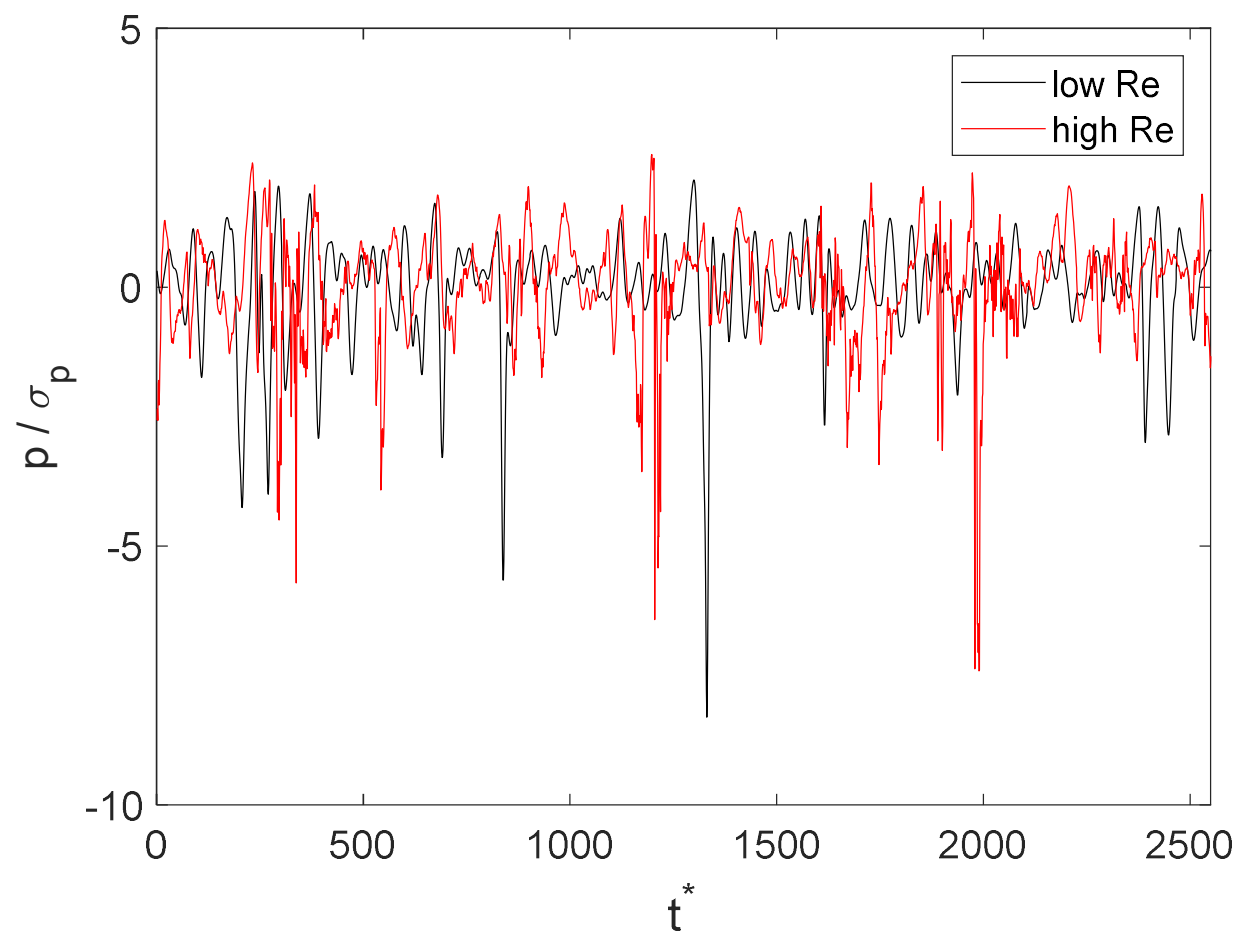

Figure 4: Pressure segments obtained at the two Re considered taken from a virtual probe located at $x / D=7$ and $r / D=1$.

The overall dependence of the pressure PDFs upon the relevant non-dimensional parameters, i.e. $x / D, r / D$ and Re, is presented in the form of 3D plots in Figures 6 and 7 for the lowest and highest Re respectively. The curves are again reported in semi-log scale and a reference Gaussian PDF is included for comparison.

The plots show that for increasing $r / D$, the region where the negative tails are relevant moves towards larger $x / D$ due to the jet flow spreading. It is confirmed that at $r / D=3$, the statistics are close to Gaussian independently from x/D and Re as an indication of the rapid decay of the hydrodynamic pressure with respect to the Gaussian acoustic fluctuations. On the other hand, the influence of pseudo-sound is more and more relevant as r/D decreases. 


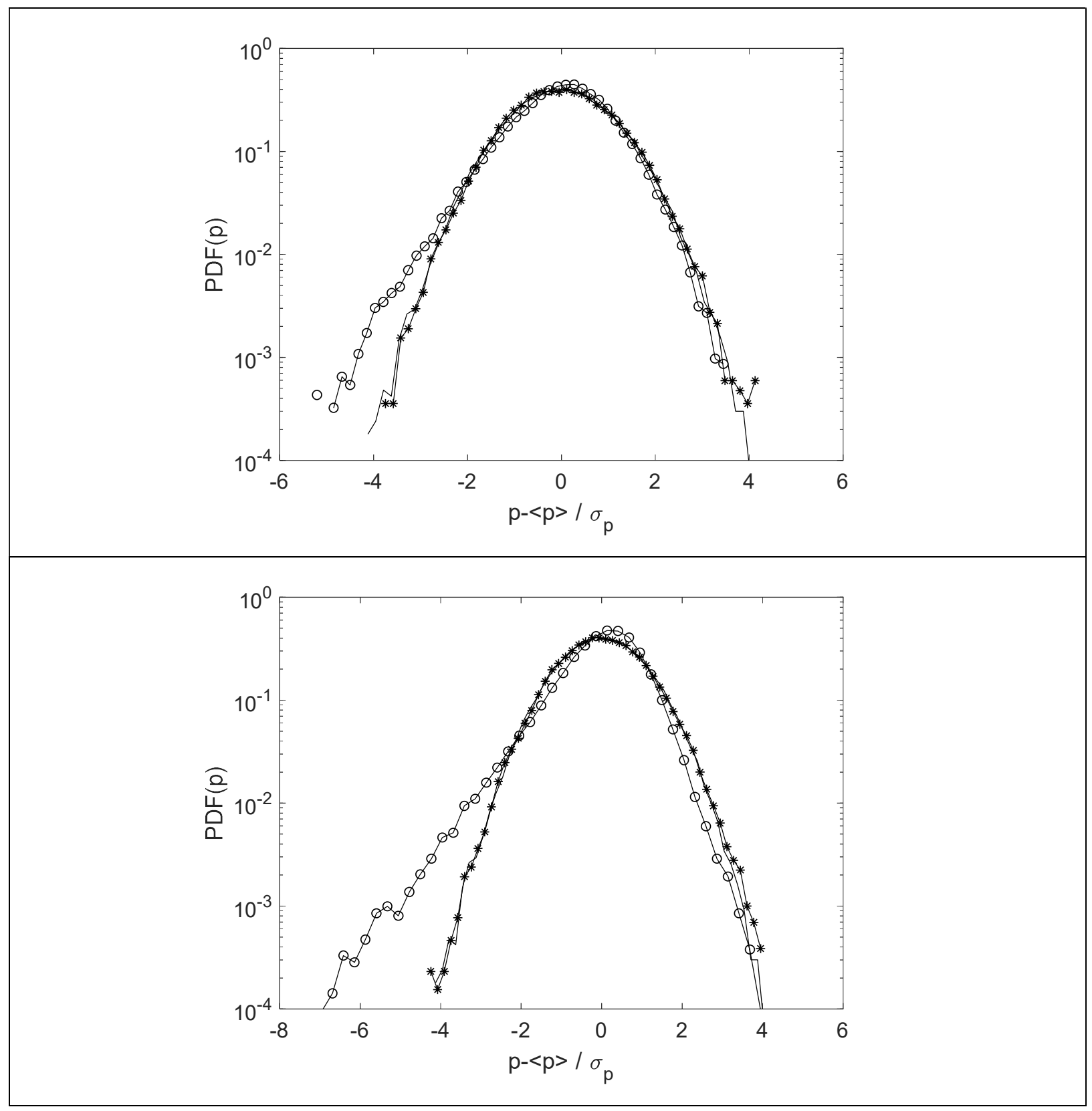

Figure 5: PDF of the pressure fluctuations for the low (top) and high (bottom) Re numbers. PDFs are computed at $x / D=6$ and for $r / D=1$ (circles) and $r / D=3$ (asterisks). The solid line is the PDF of a reference Gaussian signal obtained by a random numbers generator. 


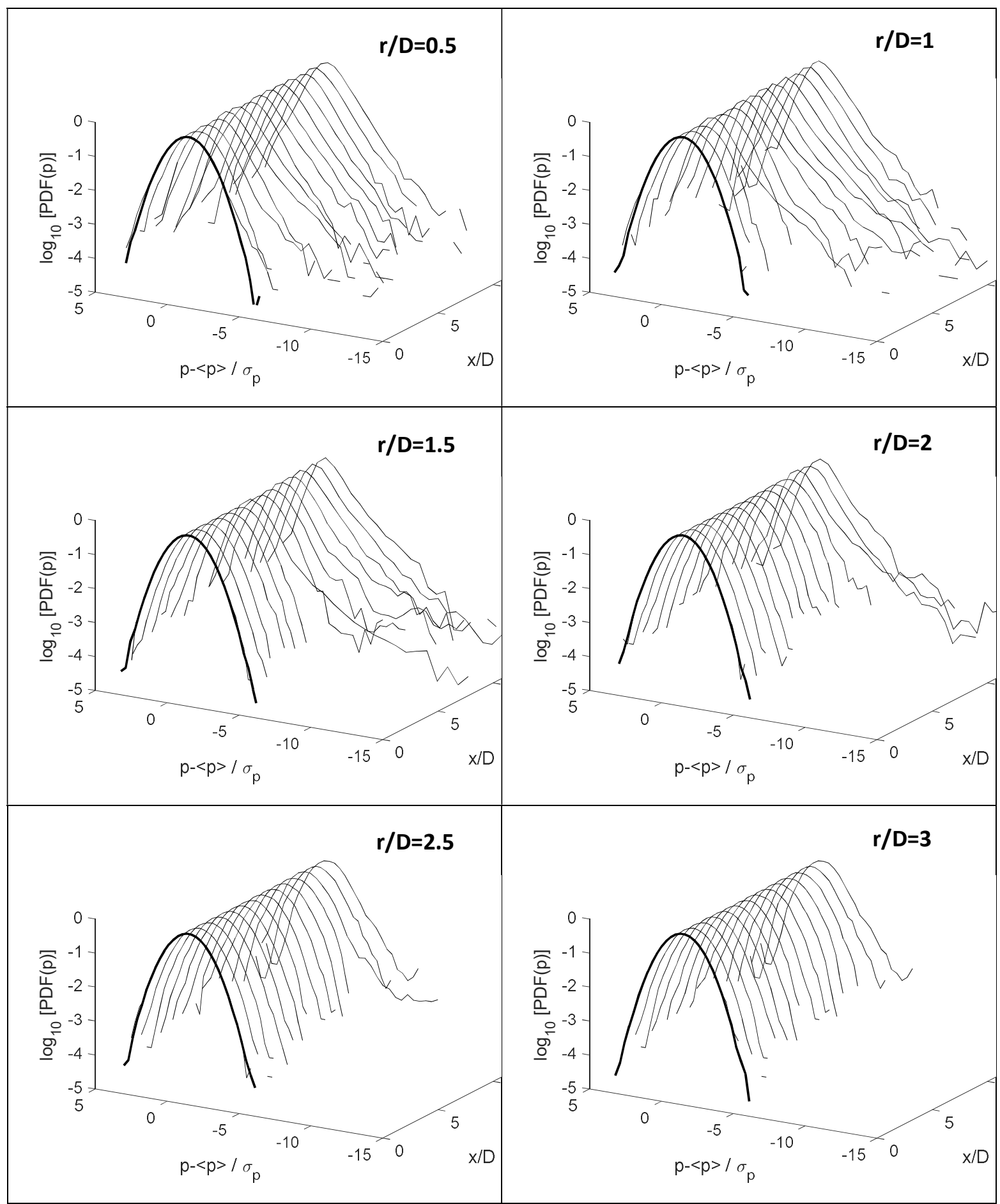

Figure 6: PDF of pressure fluctuations computed at the different r/D and at high Re. In order to better highlight the variation of the negative tails, the values of the abscissa are reversed. 


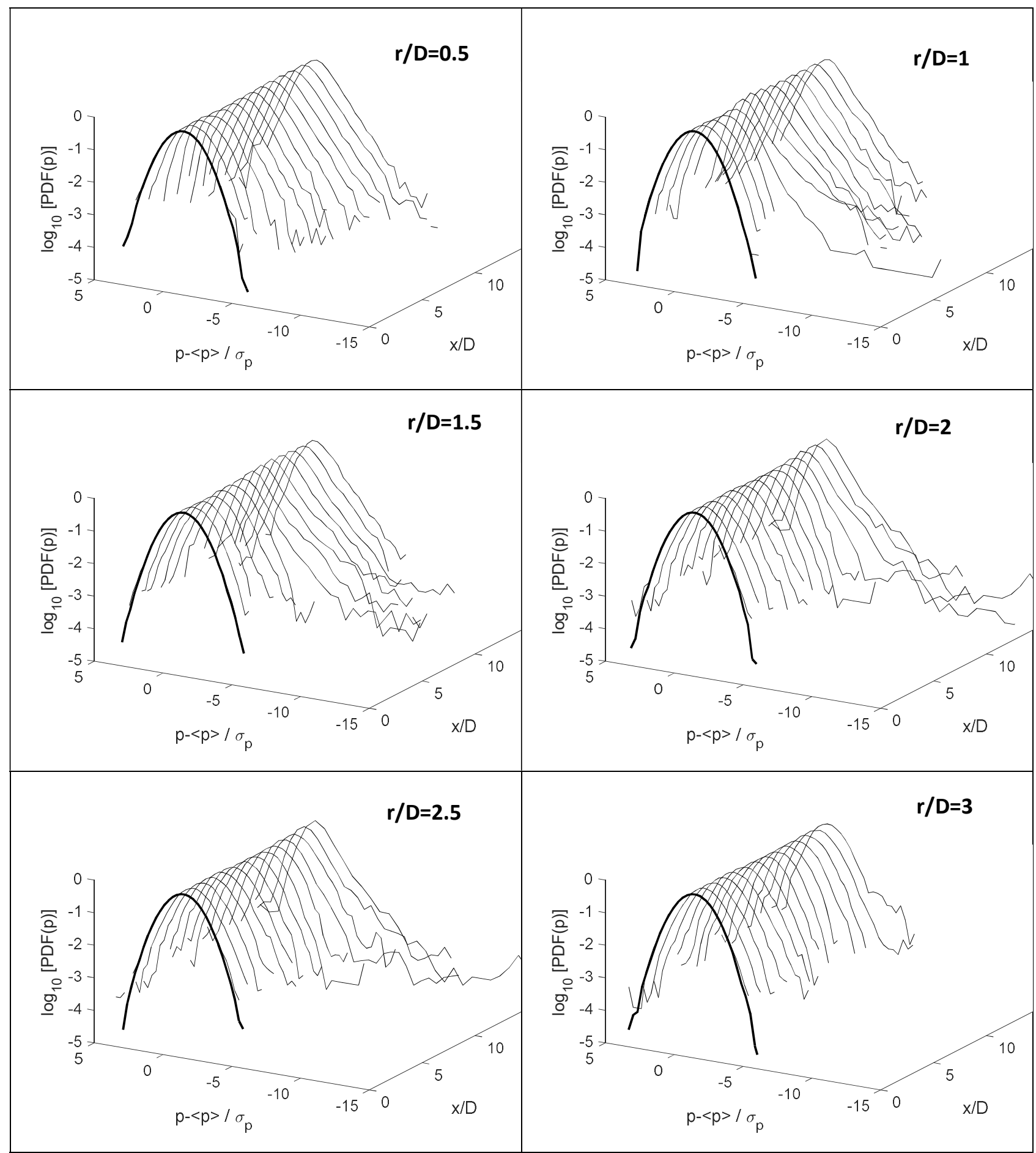

Figure 7: Same as previous figure but for the low Re case

The overall behavior is reflected in the evolution of the Flatness Factor (FF) reported in Figure 8 for both the two considered Re. The FF is presented as a function of $x / D$ and $r / D$ and the light color enhances intermittent regions identified where the FF is much larger than 3. It can be observed that for both Re, at x/D similar to or larger than the potential core length, the FF becomes much larger than 3 as a trace of the intermittent statistics. The largest peaks are observed close to the shear 
layer in correspondence of the jet lip line and remains relevant (larger than 3 ) inside the flow. It is confirmed that intermittency is not observed very close to the jet exit where hydrodynamic pulsations are relevant but their statistics are not intermittent. The most interesting result is that such a behavior is independent of Re, the only relevant difference being the lower bound of the region where intermittency becomes relevant, about $x / D=4$ at the highest $\operatorname{Re}$ and $x / D=6$ at the lowest.
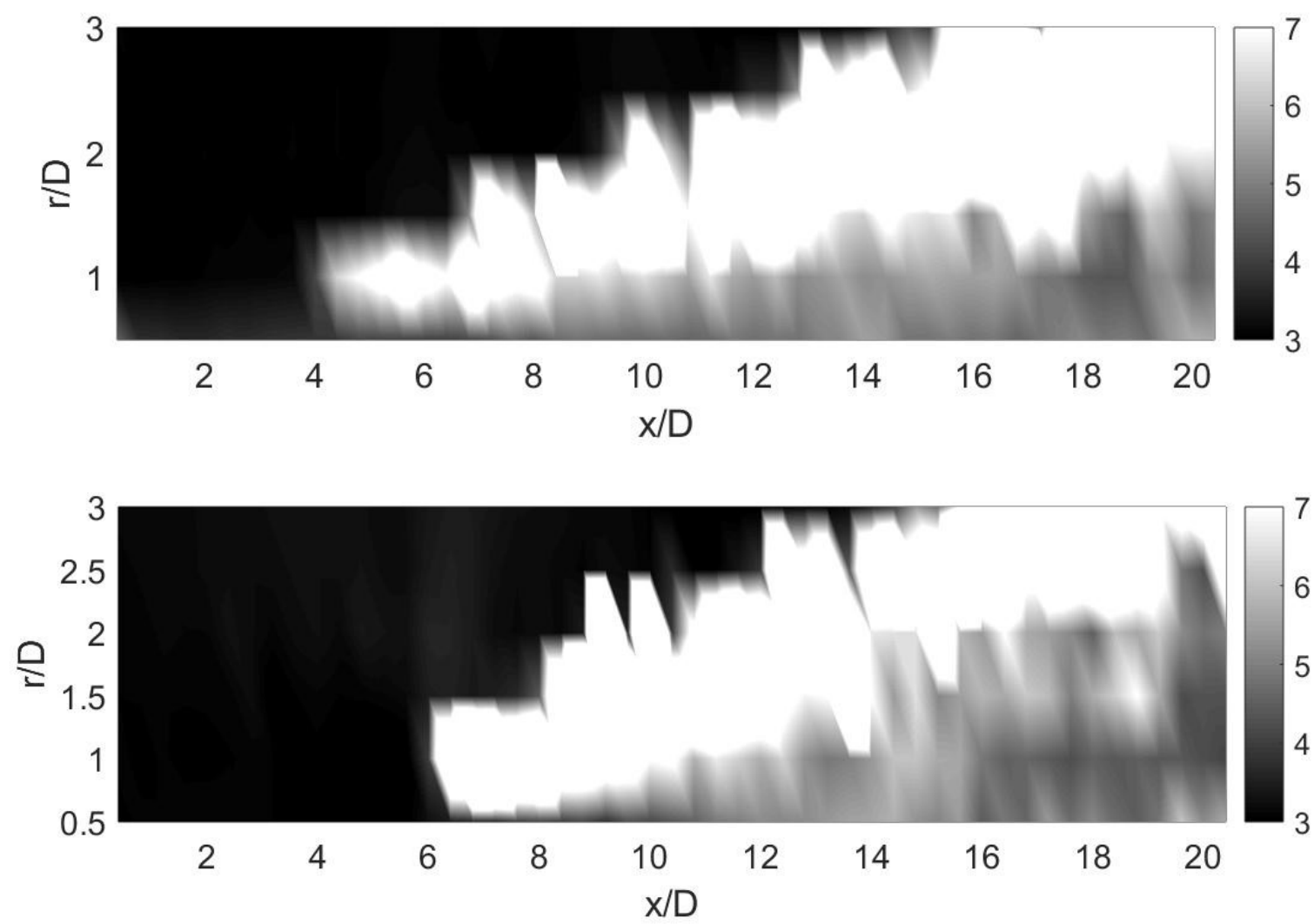

Figure 8: Flatness Factor distribution at high Re (top) and low Re (bottom). The light regions indicate amplitudes much larger than 3 and thus a high degree of intermittency.

\subsection{Intermittency of the 0-mode pressure fluctuations}

The global statistics of the 0-mode pressure is analyzed in details and the results are compared to those obtained from the original pressure signals. Examples of pressure PDFs are reported in Figure 9 along with the Gaussian reference curves for comparison. It can be observed that, in this case, intermittency is no longer observed and the statistics is very close to the Gaussian one except for small deviations of the tails at large $x / D$. This is confirmed by the spatial distribution of the FF reported in Figure 10. The amplitude is close to 3, or even lower, everywhere and no peaks are observed. Hence, it appears surprisingly that, as opposed to the statistics of the original signals, the 0 -mode pressure statistics is weakly influenced by the jet transition to turbulence and thus that the turbulence-induced intermittency seems not to influence significantly the 0-mode pressure 
fluctuations, its dynamics playing a relevant role in the noise generation mechanism. This behavior can be due to the fact that the short-term negative pressure drops responsible for the deviation from Gaussianity may be associated with the passage of local vortex structures having a small correlation length in the azimuthal direction and thus inducing an effect in the azimuthal harmonics that is not statistically relevant when estimated through the traditional indicators. This interpretation has been verified by the estimation of the FF for higher-order modes that results to be close to 3 as well (results are not reported for brevity). Nonetheless, we shall see that the wavelet decomposition will be able to reveal that an intermittent content indeed exists also for the 0 -mode but only at certain frequencies.
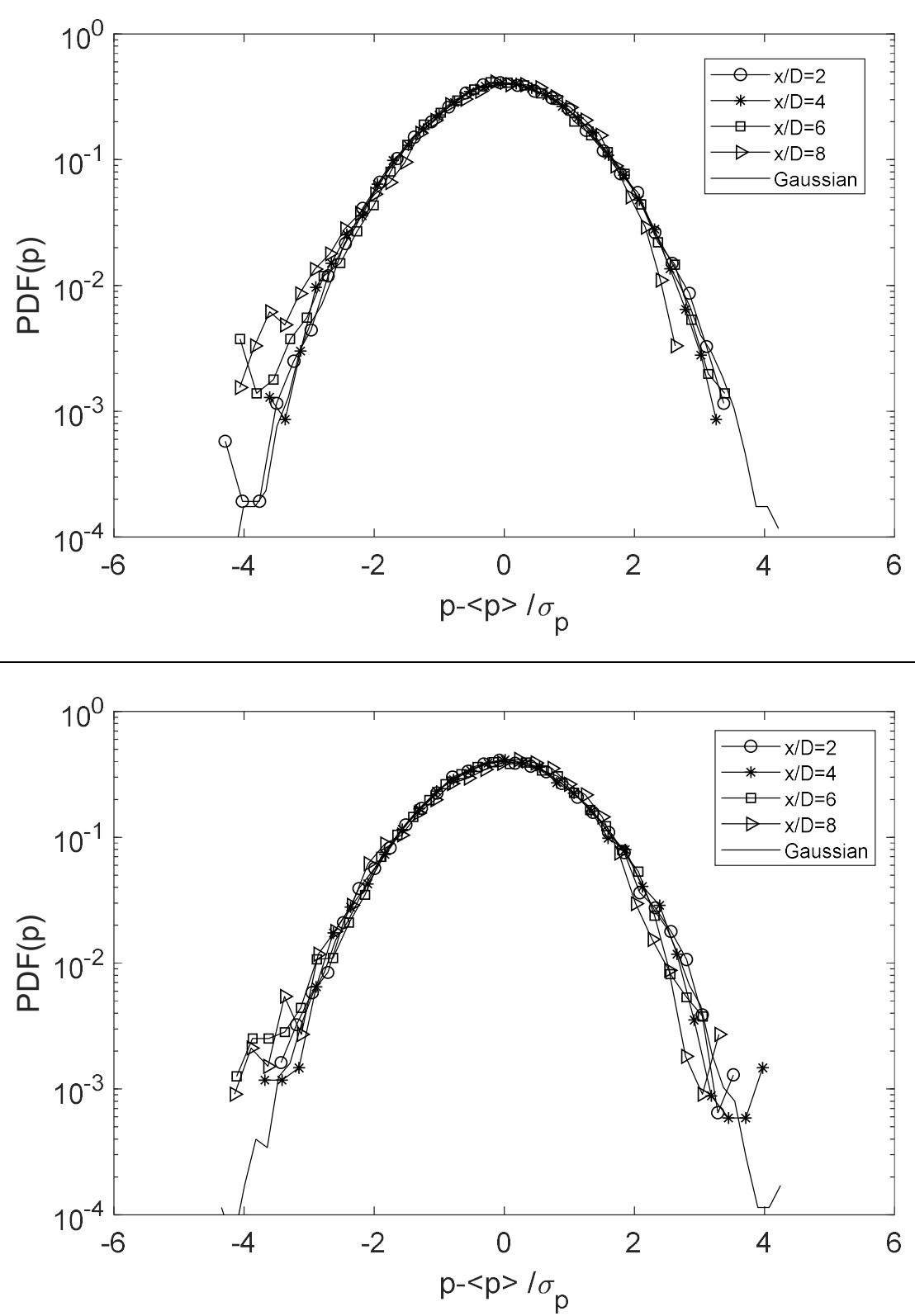

Figure 9: PDF of the mode-0 pressure fluctuations at the low (top) and high (bottom) Re computed at $r / D=1$ for different axial positions. The solid line is the PDF of a reference Gaussian signal obtained by a random numbers generator. 

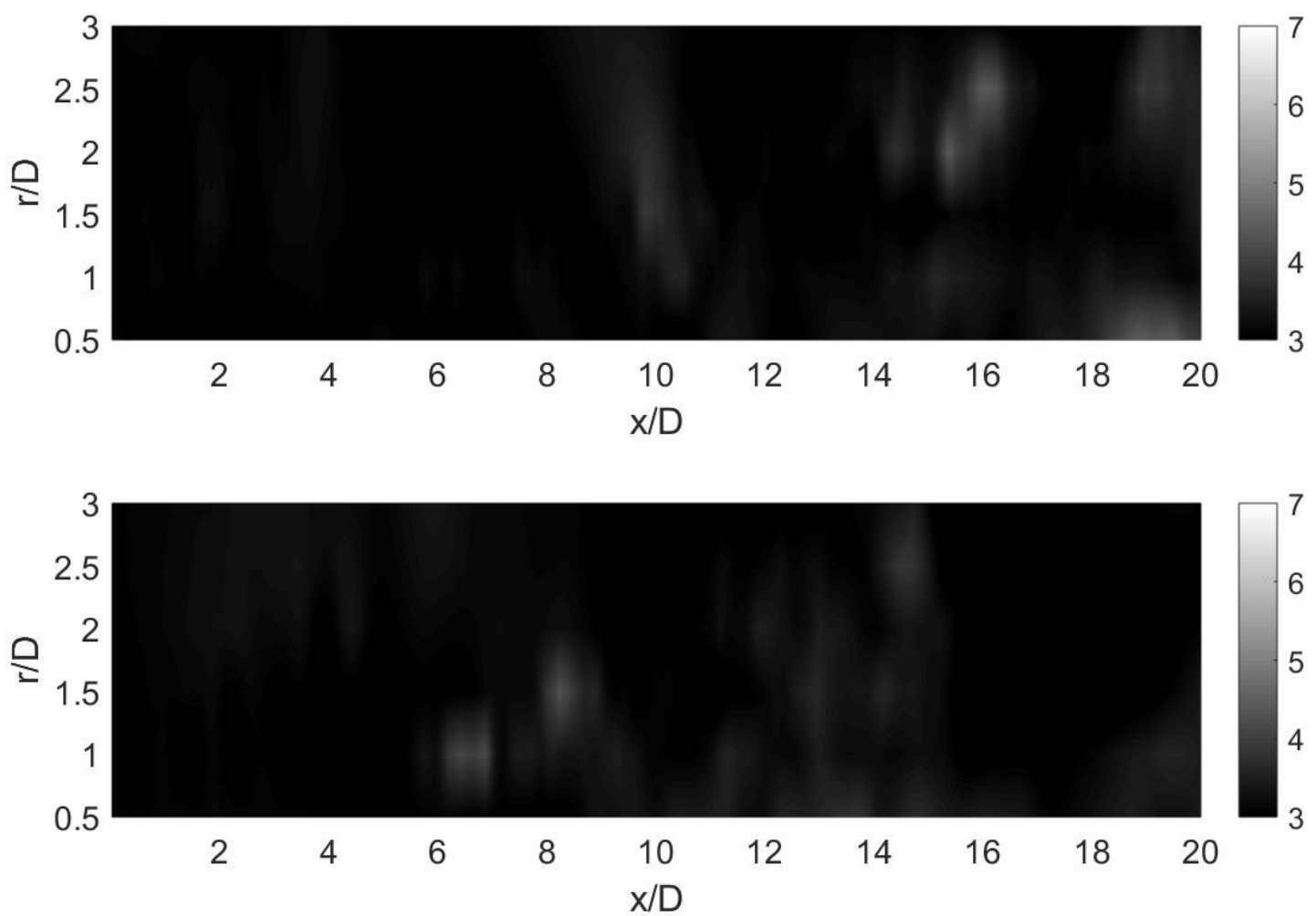

Figure 10: Flatness Factor distribution at high Re (top) and low Re (bottom) computed from the mode-0 pressure signals.

The above results suggest that contributions to intermittency from all frequencies are mixed in the flatness factor standard estimation. Low frequencies that possess significant energy content downstream of the end of the potential core might not necessarily be those that display significant intermittency. The wavelet analysis can overcome this limitation by providing an estimation of intermittency scale-by-scale. The idea to be exploited is that intermittency is contained to a certain range of frequencies associated to the Kelvin-Helmholtz instability and the use of the wavelet transform allows for the identification of this behavior through the calculation of a frequency dependent FF, denoted as WFF and defined in Sec. 2. The representation of WFF as a function of St is an efficient way to highlight the statistical content of a signal when intermittency is confined at a specific frequency. This is the case of the signals analyzed therein taken in the near-field region of the jet. Examples are reported in Figure 11 where the WFF is computed at $r / D=1$ and $x / D=2$, an axial position where, according to the previous analysis no intermittency is present. It can be observed that in all cases (low and high Re, original and 0-mode signals) the WFF peaks at St around 0.4 that is close to the $\mathrm{St}_{\mathrm{KH}_{\mathrm{H}}}$ determined from the PSD. It is also observed that the 0-mode pressure exhibits a significant degree of intermittency as opposed to the global results discussed above. Furthermore, the cases at low Re are characterized by a larger amplitude of WFF with respect to the high Re as a trace of a larger local intermittency. As stated above, "global" intermittency refers to statistical 
moments and PDFs that contain mixed information about all the frequencies, whereas the WWF is analysed in "localised" frequencies.

An overall representation of the 0 -mode intermittent content is given in Figure 12 where WFF as a function of St is reported for different $x / D$ position. This plot highlights the regions where local intermittency is relevant. In the low Reynolds number case, a very high local intermittent degree is observed close to the jet exit in the potential core region. The peaks are concentrated around the Kelvin-Helmholtz frequency and decay rapidly for $x / D$ larger than about 7 . The behavior observed at higher $\mathrm{Re}$ is somewhat smoother. The local intermittent degree remains large even outside of the potential core even though the largest levels are reached at $x / D$ lower than 5 . It is also interesting to note that the frequency where the maximum WFF is reached is more dependent upon $x / D$ this behavior being in agreement with the evolution of the energy bump observed from the power spectra (see Figure 3a). Similar results are obtained in other radial positions and are not reported here for brevity.

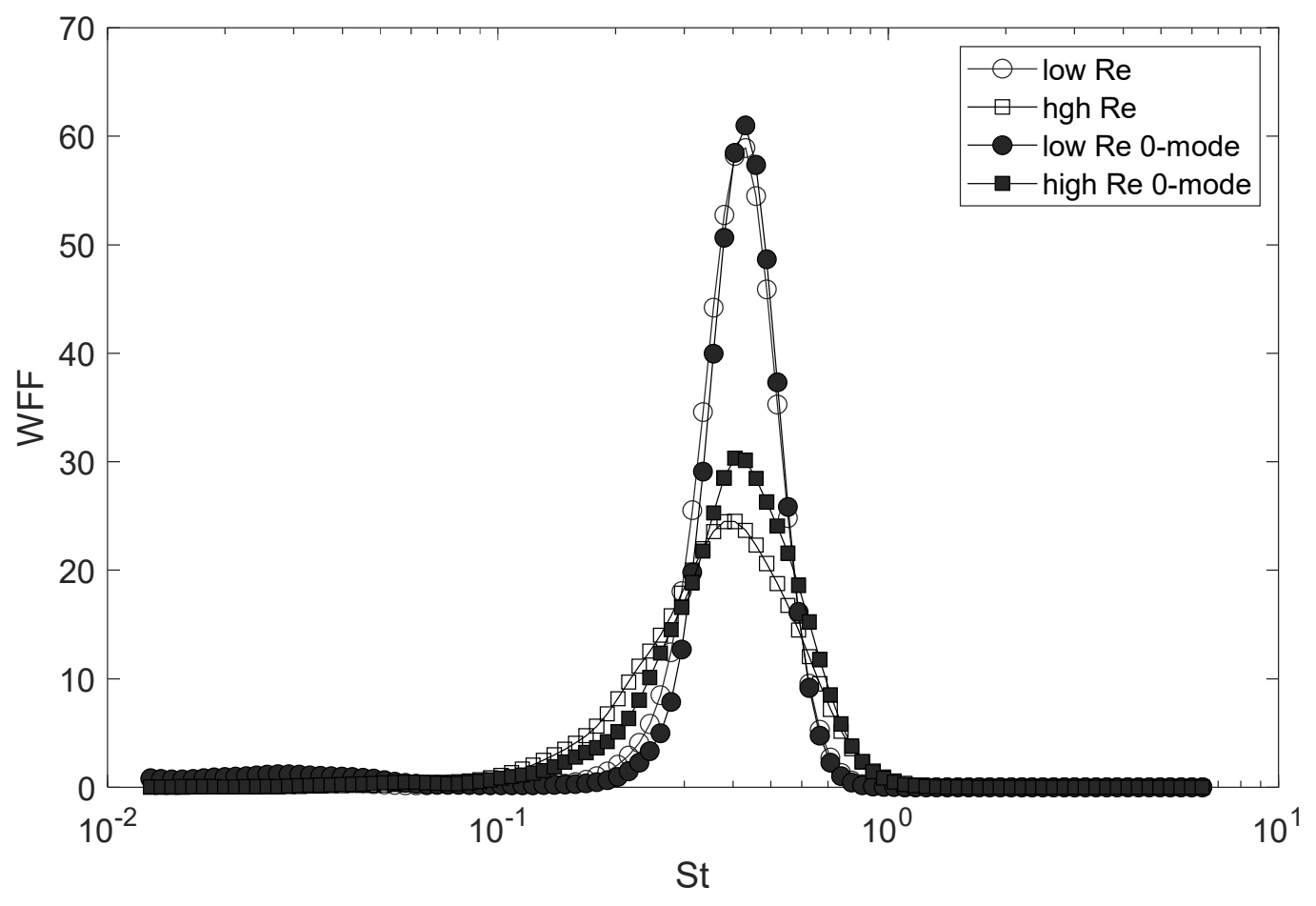

Figure 11: WFF computed at $x / D=2$ and $r / D=1$ 


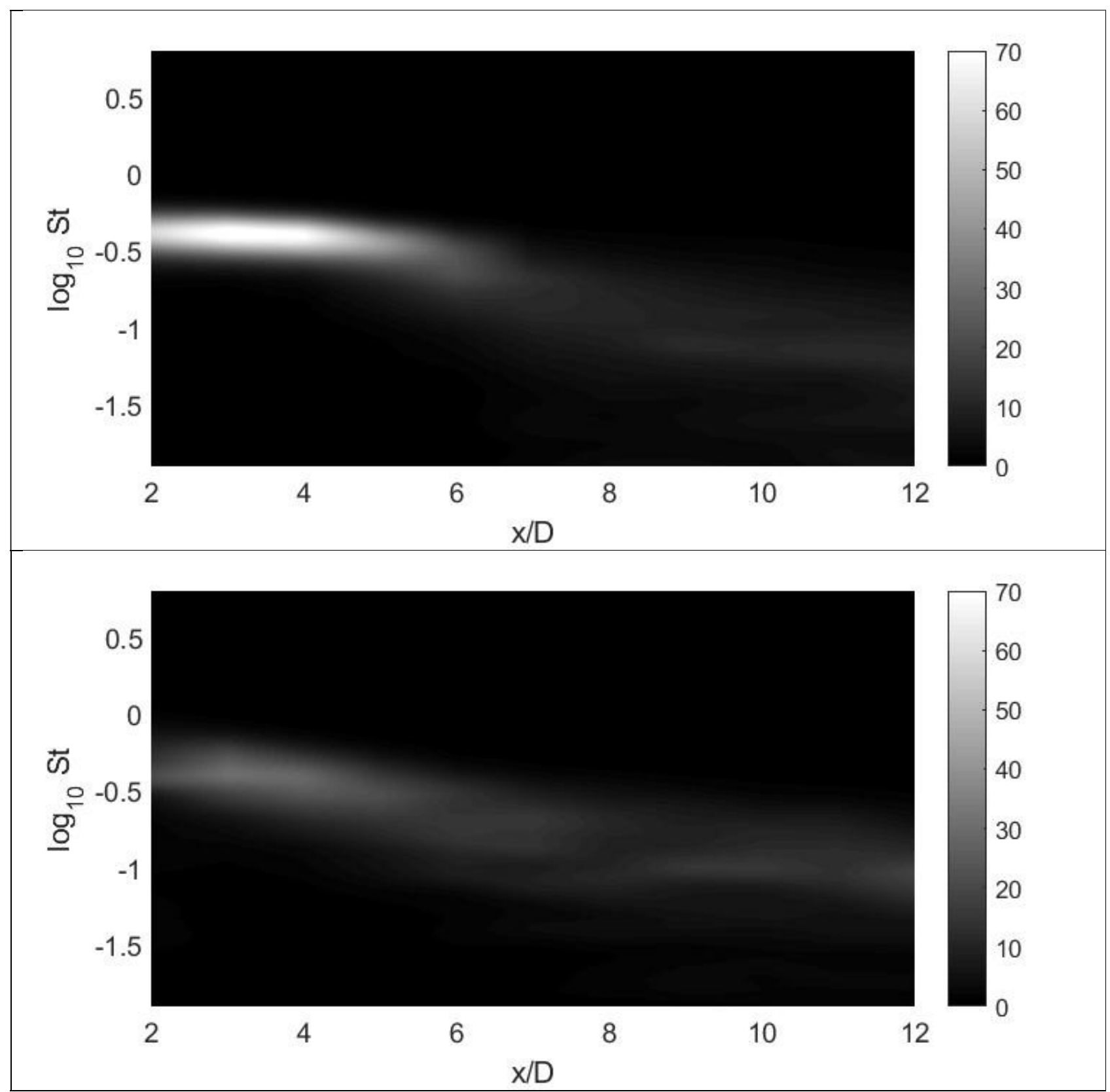

Figure 12: WFF as a function of St computed for the 0-mode pressure signals: (a) low Reynolds number at $r / D=1 ;(b)$ high Reynolds number at $r / D=1$. The amplitude scale is the same adopted in Figure 11. 


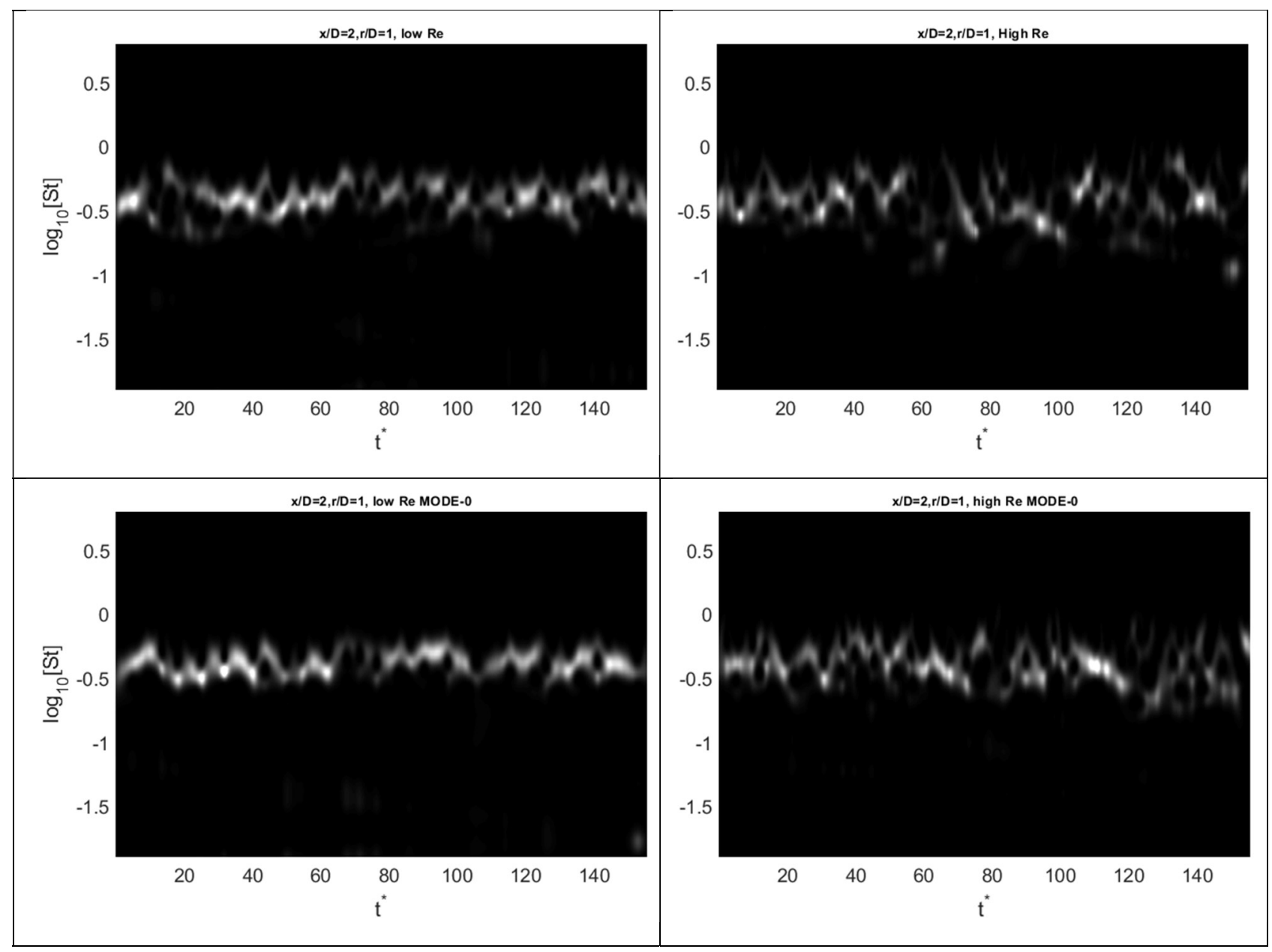

Figure 13: LIM2 computed from segments of pressure signals taken at $x / D=2$ and $r / D=1$. For each plot the gray scale ranges from 0 to 150. From upper right to lower left plot: Low Reynolds, High Reynolds, Low Reynolds 0-mode, High-

Reynolds 0-mode

The intermittent behavior can be qualitatively verified by the evolution in time of the FF, retrieved by the LIM2. Segments of LIM2 extracted from the same signals analyzed previously are given in Figure 13. It can be clearly observed that in all cases, the largest intermittent events are concentrated around the Kelvin-Helmholtz mode. It is notably to observe that also for the 0 -mode the evolution of LIM2 clearly exhibit large peaks (even larger than 150) with an amplitude that is even larger than that obtained in the original signals. Again, we underline that this behavior was completely missed by the analysis of the global intermittency indicators shown above.

The results obtained so far are in agreement with the two points coherence measurements by Jaunet et al. [10]. Their coherence lengths educed from the 0 -th modes of the velocity field are shown to be much higher than those of the full signal in a broad frequency range. This confirms that that axisymmetric events associate to the Kelvin-Helmholtz instability do present a significant degree of intermittency and most of the difference between the intermittency of the full signal and that of the 0 -th mode is contained in frequencies associated to the Kelvin-Helmholtz instability. 
The conditional statistics is retrieved by the application of the procedure outlined in Sec.2 based on the selection of events from the maxima of the LIM2. On the basis of the previous analyses, it makes sense to apply the same procedure to the mode 0 signals and to analyze the conditional statistics also for those cases comparing them with the statistics of the original signals.

An interesting indicator that can be retrieved is the mean frequency of the intermittent event (denoted as MIF in Sec. 2) that can be retrieved by averaging the wavelet scale $\tilde{s}$ of the set of coefficients selected by the conditional procedure. This quantity is compared with the frequency of the Kelvin-Helmholtz mode. Figure 14 shows an example of the MIF variation as functions of $x / D$ for a selected case $(r / D=1)$ at low and high $R$. The result obtained from the 0 -mode pressure signals is also included. It is shown that the MIF value decays for increasing $x / D$ and remains similar to that of the Kelvin-Helmholtz frequency retrieved from the maxima of the power spectra (see Figure 3 ). The amplitudes are similar in the different cases even though the MIF corresponding to the 0-mode are slightly larger than those retrieved from the original signals. These results have been confirmed at the other radial positions and are not reported here for brevity.

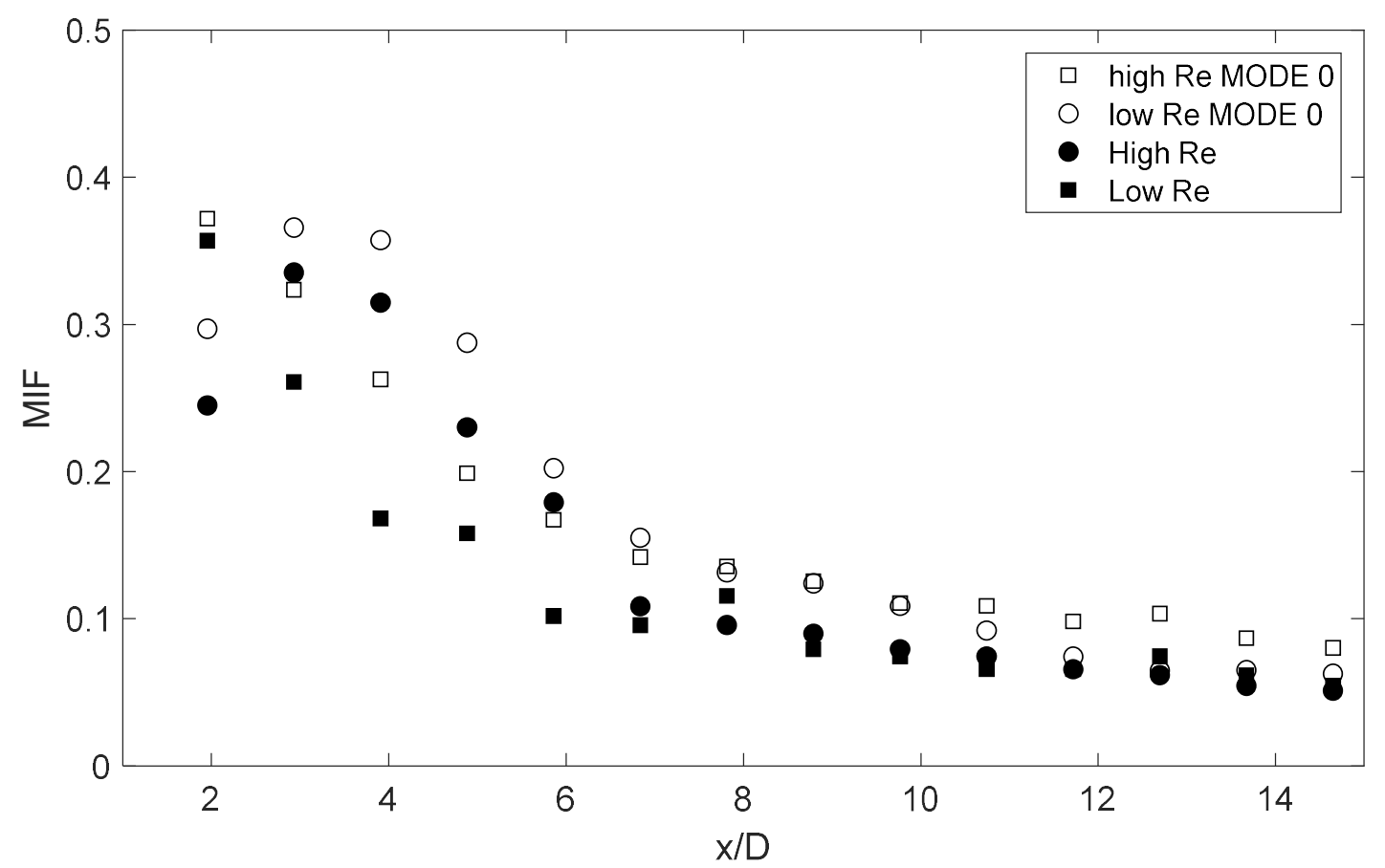

Figure 14: MIF computed at $r / D=1$ for the original (filled symbols) and the 0 -mode pressure signals (empty symbols).

Once the set $\{\tilde{s}, \tilde{t}\}$ of scale and time of the selected events is available, it is possible to compute the PDF of the two main quantities outlined in Sec. 2, i.e.:

- Events energy: $\quad P D F\left[w(\tilde{s}, \tilde{t})^{2}\right]$

- Events time delay: $P D F[\Delta t]$

These quantities are computed for both the original and the mode- 0 pressure signals and descriptive results are reported in the following in the standard normalized form.

The PDFs of the events' energy are presented in Figures 15-16-17 for $r / D=1,2$ and 3 respectively and both $\mathrm{Re}$. In each plot, the results obtained at reference $x / D$ positions are presented. The 
notation $w^{2^{*}}$ (and $\Delta t^{*}$ in the successive figures) indicates the use of normalized variables obtained by subtracting the mean value and then dividing by the standard deviation.

According to [36] and [37] the PDFs exhibit a clear exponential positive tail that can be approximated by a pure exponential function. At the largest $r / D$, events with a lower level of energy become statistically relevant and a quasi-Gaussian decay of the negative tail is observed [36]. To the extent of the most energetic events (larger than the mean), the shape of the PDF remains basically the same for the whole set of $x / D$ and $r / D$ considered. In addition, the mode-O PDFs look very similar and the effect of Re is weak. The observed differences have to be attributed to a lack of statistical convergence that occurs at low Re and for the 0-mode cases as an effect of the lower number of events selected. However, from the physical viewpoint, the achieved results seem universal. For $r / D>1$, the selected events include low energy amplitudes and the PDFs are more similar to the lognormal shapes reported in [37].

Similar outcomes are obtained in the analysis of the time delay $\Delta t$. The PDFs for $r / D=1,2$ and 3 and both $\mathrm{Re}$ are presented in Figures 18-19-20. Again, a few reference $\mathrm{x} / \mathrm{D}$ positions are selected and results are compared to each other. Also in this case, the positive tail can be approximated by an exponential decay law event though at the lowest Re and small $r / D$ the statistical convergence is poor and the exponential approximation applies only qualitatively. In the other cases, the pure exponential approximation applies well and, as for the events energy amplitude presented above, the dependence upon the governing parameters ( $x / D, r / D$ and $R e)$ is weak for both the original and the 0 -mode signals.

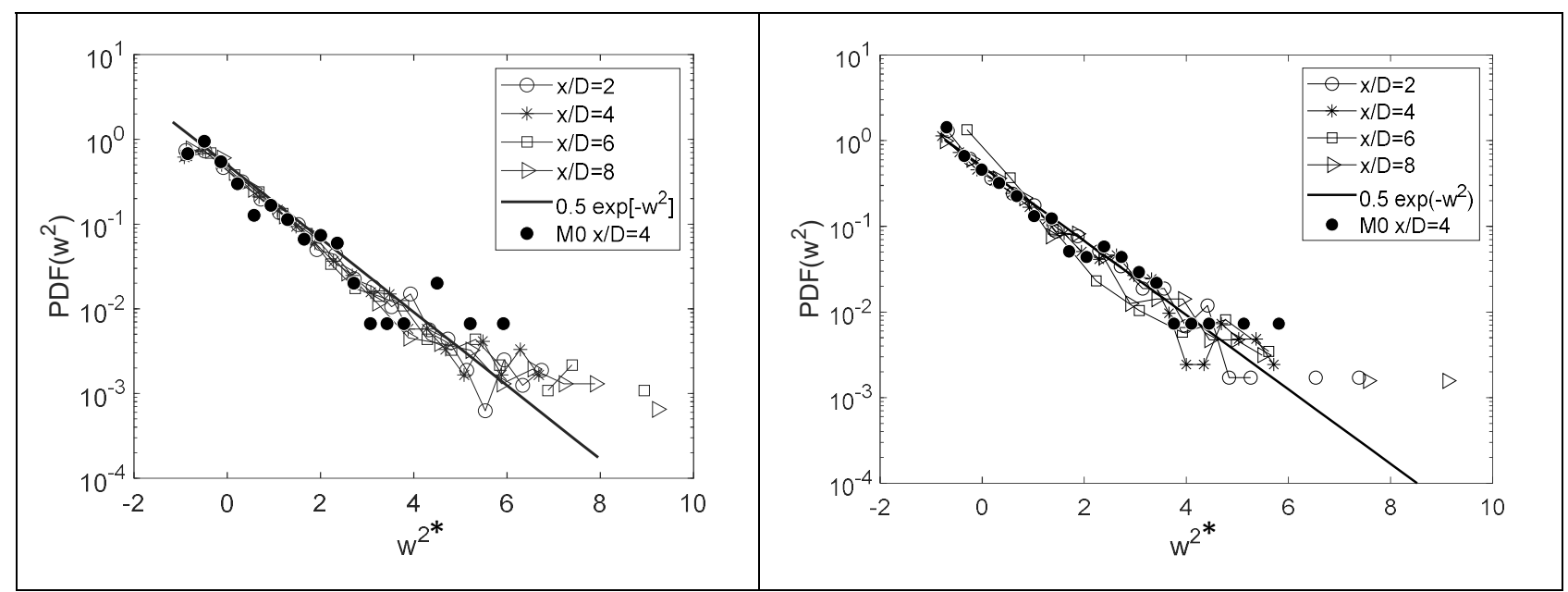

Figure 15: PDF of the square of wavelet coefficients selected in the conditioning procedure. The cases reported correspond to high (left) and low (right) Re at $r / D=1$ 

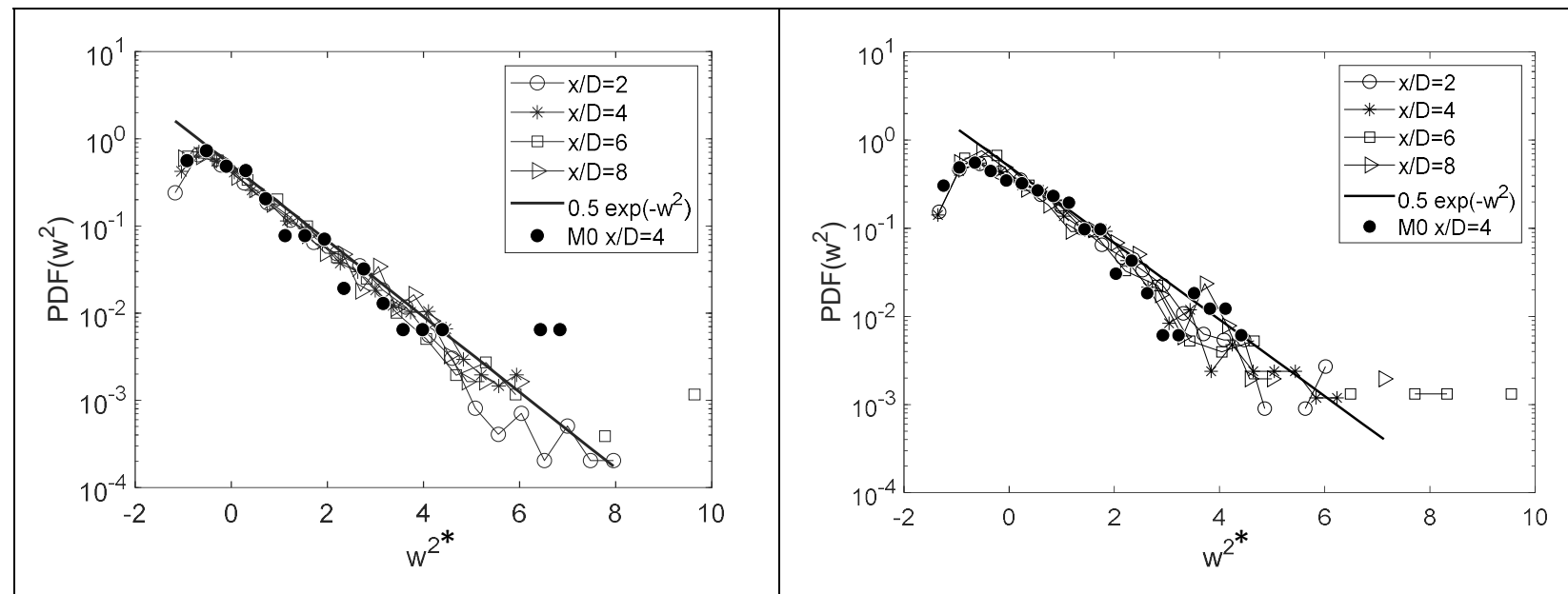

Figure 16: Same as previous plot at $r / D=2$

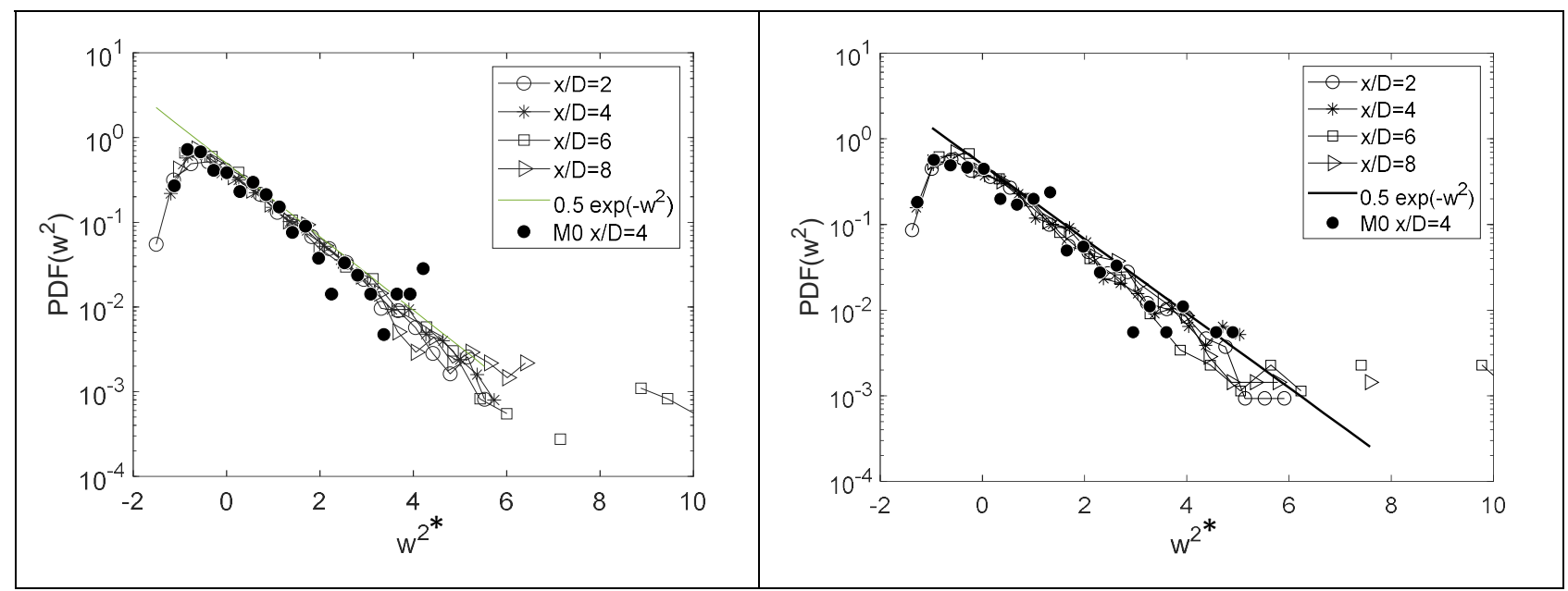

Figure 17: Same as previous plot at $r / D=3$
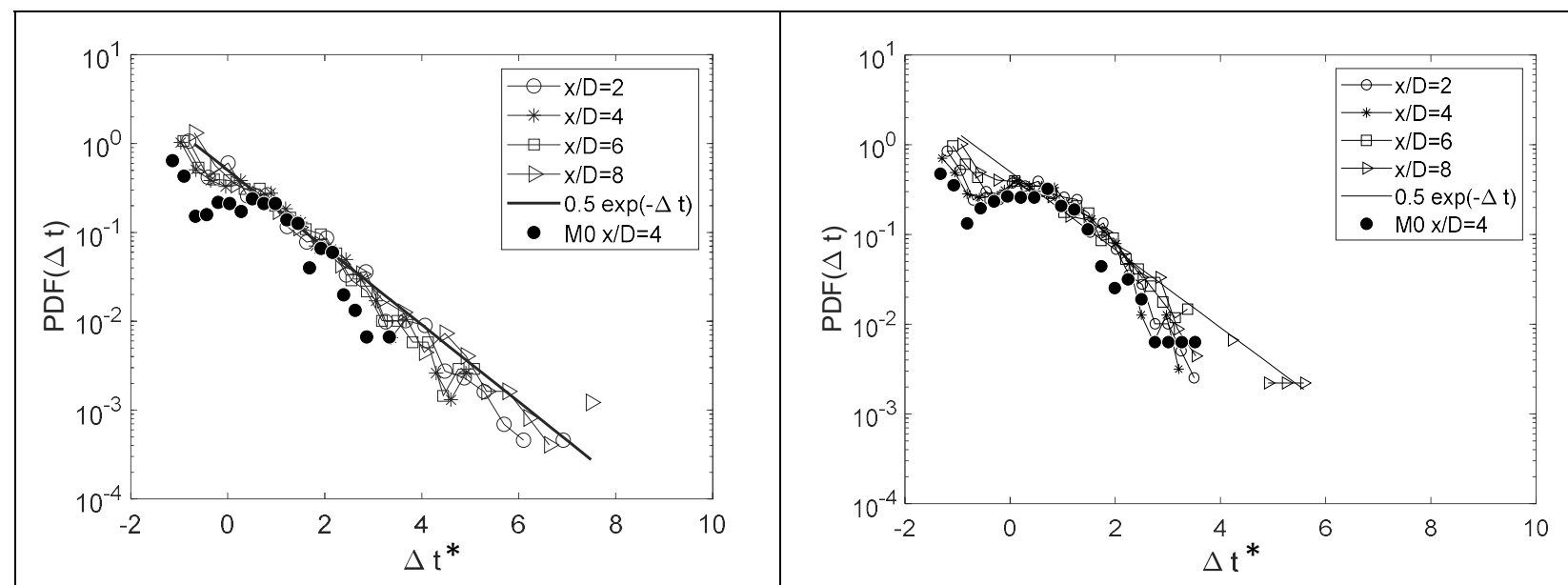

Figure 18: PDF of the time delay between successive events $(\Delta t)$. The cases reported correspond to high (left) and low (right) Re at $r / D=1$ 


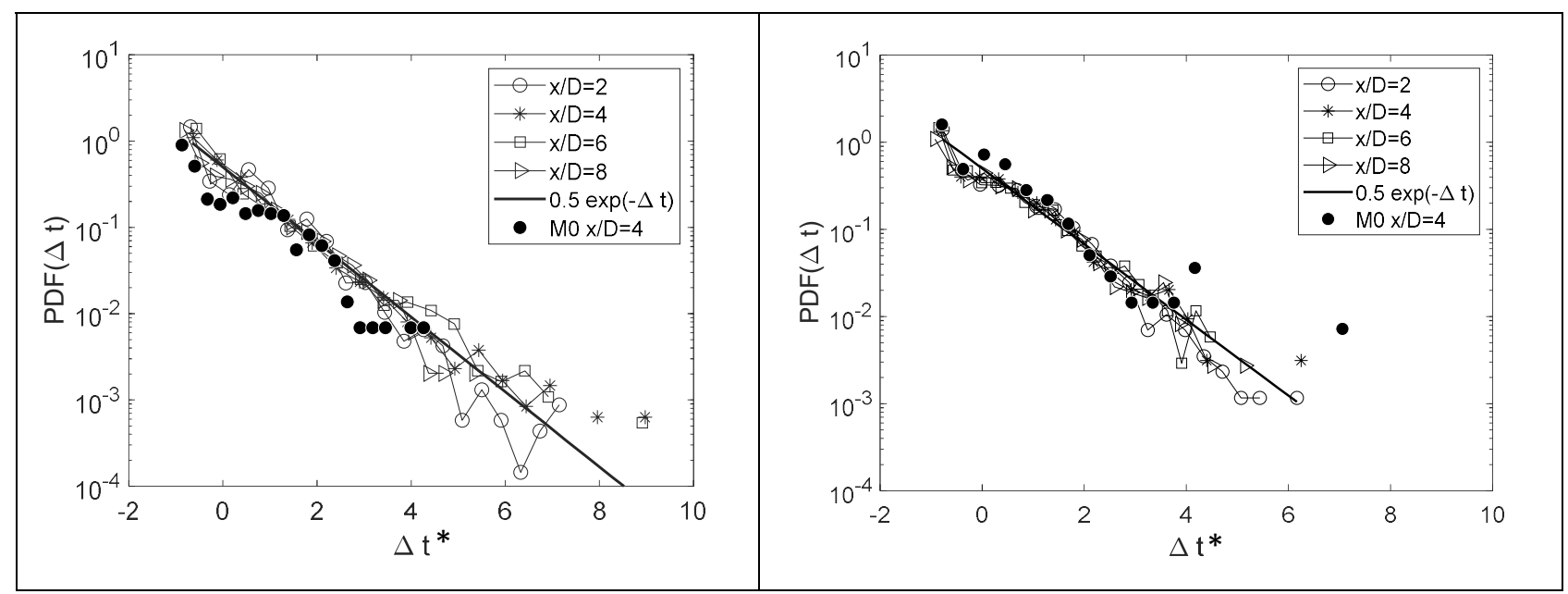

Figure 19: Same as previous plot at $r / D=2$

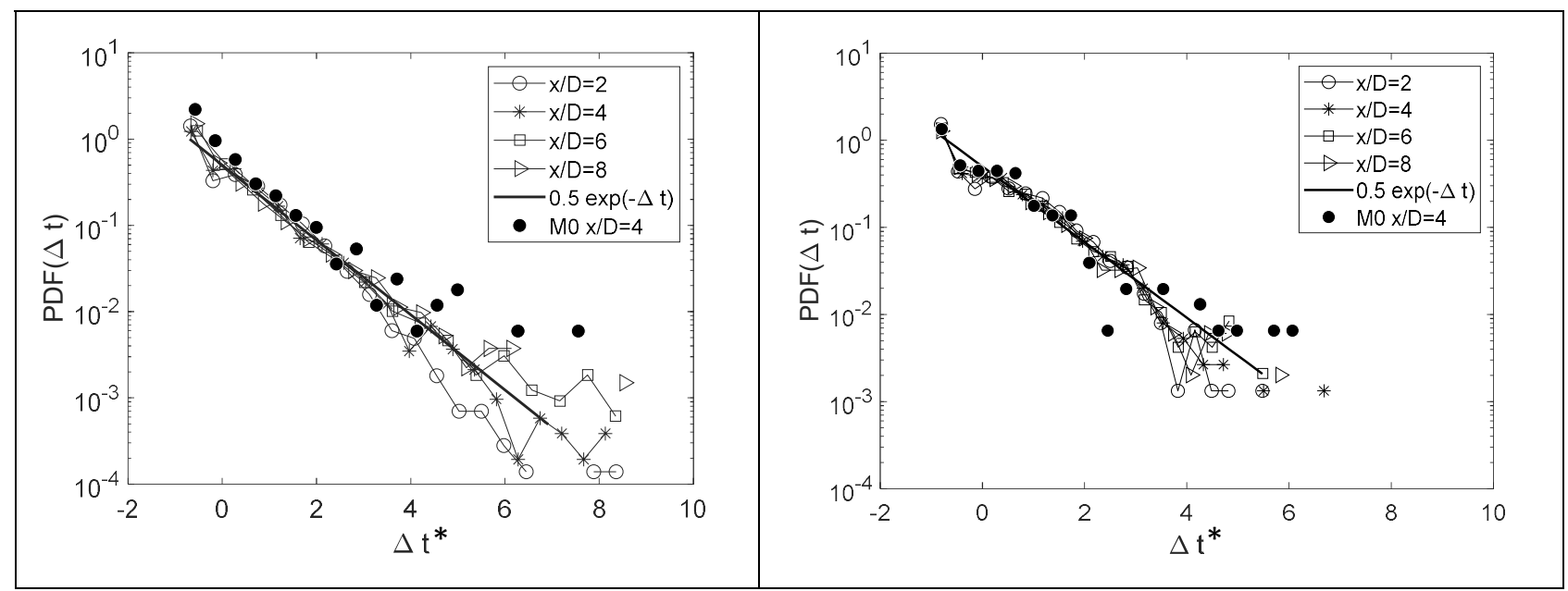

Figure 20: Same as previous plot at $r / D=3$

The universal behavior outlined above justifies the search for a stochastic model based on proper analytical approximations of the measured PDFs of both the energy and the waiting time. Relatively complex functional forms have been proposed by Camussi et al. [36-37] where results very similar to the present ones have been obtained. However, to the purpose of providing a model as simple as possible, we have observed that a pure exponentially decaying function can provide a reasonably good approximation for all the analyzed cases.

The following analytical expression can therefore be assumed:

$$
P D F(X)=0.5 e^{-X}
$$

where $X$ is the random variable (energy or time delay).

It has to be pointed out that the exponential PDF typically governs Poisson stochastic processes characterized by a lack of memory and frequently governs the waiting time statistics. Indeed, as pointed out by Feller [57], for a phenomenon characterized by a complete lack of memory (or aging), the probability distribution of the duration must be geometric or exponential. The memoryless property associated to the exponential distribution is expected for the waiting time statistics but it 
is less trivial to be interpreted for the energy. It appears that the flow structures underlying the selected events are convected by the flow and remain statistically independent in terms of their dynamics even though their typical length-scale is large (as is the case of the wavepackets, see e.g. [58] and [59]). A definite clarification of this aspect can be achieved by determining the topology and physical nature of the selected events, an issue that can be addressed by conditional analyses of kinematic quantities (3D velocity fields or vorticity, see e.g. the recent papers [60-62]), a challenging task that is left for future studies.

\section{Conclusions and final remarks}

The pressure fluctuations in the near field of two compressible jets at $M=0.9$ and quite different $\operatorname{Re}$ are analyzed in detail to highlight intermittency through the estimation of proper statistical indicators. The data are obtained numerically in a region spanning 20 diameters in the axial and 3 diameters in the radial direction. The pressure data are decomposed azimuthally and the statistical properties of the 0-mode component are analyzed in details in order to highlight properties that can be directly correlated to the generation of noise. The mode- 0 analysis represents the main step forward with respect to the state of the art along with the evaluation of the Reynolds number effects and the corresponding stochastic modelling.

The analysis of intermittency is carried out using two approaches: global and local. The global intermittency analysis is based on the computation of standard statistical quantities (the PDFs and the Flatness Factor) of either the original or the reconstructed 0 -more pressure signals. It is observed that pressure exhibits a relevant (global) intermittent non-Gaussian statistics in the region downstream of the potential core and across the shear layer. The spatial distribution of the intermittent statistics seems to suggest that the deviation from Gaussianity is relevant outside the potential core, this behavior being weakly affected by the Reynolds number.

Interesting features are achieved when the 0-mode pressure components are analyzed. In this case the intermittent content is revealed through the local intermittency analysis that is carried out by the application of the wavelet transform, this procedure allowing for a localized counterpart of the Flatness Factor to be retrieved. The analysis is focalized upon the statistical properties of the pressure fluctuations at the typical Kelvin-Helmholtz frequency. Conditional statistics are recovered by selecting intermittent events on the basis of a wavelet-based local Flatness Factor (called LIM2) and by extracting their energy and time of appearance. In the local framework, the degree of intermittency of the 0 -mode case is relevant and it is significant also within those regions of space where the global intermittency of either the original and the 0 -mode signals was negligible.

The conditional PDFs of both the energy and the waiting time between successive intermittent events are very similar to each other even though they appear. to be affected by the statistical convergence of the data selected on the basis of the LIM2 criterion and further investigations are needed to verify this outcome. To this extent, a larger number of samples (for example delivered from a dedicated experiment) would provide a definite assessment of the proposed model. Even though qualitatively, it is anyway observed that the PDFs are weakly affected by the flow parameters and can be modeled by a pure exponential distribution.

The achieved results seem to suggest that the dynamics of the noise emitting structures is intrinsically correlated to their evolution governed by the Kelvin-Helmholtz instability mechanism and by the intermittent statistics associated to it. In this "local" sense, intermittency plays a relevant 
role from the viewpoint of the generation of noise. The connection between local and global intermittency with noise emission is a key issue that surely deserves further investigations and remains a task for future studies.

\section{References}

[1] Lighthill, M.J.: On sound generated aerodynamically. I. General theory. Proc. R. Soc. A Math. Phys. Eng. Sci. 211, 564-587 (1952)

[2] Lighthill, M.J.: On sound generated aerodynamically. II. Turbulence as a source of sound. Proc. R. Soc. A Math. Phys. Eng. Sci. 222, 1-32 (1954)

[3] Mollo-Christensen, E., Koplin, M.A., Martuccelli, J.R.: Experiments on jet flows and jet noise far-field spectra and directivity patterns. J. Fluid Mech. 18, 285-301 (1964)

[4] Tam, K.W., Golebiowski, M., Seiner, J.M.: On the two components of turbulent mixing noise from supersonic jets. AIAA Paper 96-1716 (1996)

[5] Karabasov, S.A.: Understanding jet noise, Phil. Trans. R. Soc. A 368, 3593-3608 (2010)

[6] Tam, C.K.W., Viswanathan, K., Ahuja, K.K., Panda, J.: The sources of jet noise: experimental evidence. J. Fluid Mech. 615, 253-292 (2008)

[7] Jordan, P, Colonius, T.: Wave Packets and Turbulent Jet Noise. Annual Review of Fluid Mechanics. 45, 173-195 (2013)

[8] Crow, S. C. 1972 Acoustic gain of a turbulent jet. In Phys. Soc. Meeting, Univ. Colorado, Boulder, Paper IE, vol. 6.

[9] Crighton, D. G. \& Huerre, P. 1990 Shear-layer pressure fluctuations and superdirective acoustic sources. J. Fluid Mech. 220, 355-368.

[10] Cavalieri, A. V. G., Jordan, P., Colonius, T. \& Gervais, Y. 2012 Axisymmetric superdirectivity in subsonic jets. J. Fluid Mech. 704, 388-420.

[11] Léon, O. \& Brazier, J. -P. 2013 Investigation of the near and far pressure fields of dualstream jets using an Euler-based PSE model. In 19th AIAA/CEAS Aeroacoustics Conference and Exhibit.

[12] Sinha, A., Rodrìguez, D., Brés, G. A. \& Colonius, T. 2014 Wavepacket models for supersonic jet noise. J. Fluid Mech. 742, 71-95.

[13] Cavalieri, A.V.G., Rodríguez, D., Jordan, P., Colonius, T., Gervais, Y.: Wave packets in the velocity field of turbulent jets. J. Fluid Mech. 2013; 730:559-592. 
[14] Zhang, M., Jordan, P., Lehnasch, G., Cavalieri, A.V.G., Aggarwal, A.: Just enough jitter for jet noise?. AIAA Paper 2014-3061 (2014)

[15] Tissot, G., Zhang, M., Lajus Jr, F.C., Cavalieri, A.V.G., Jordan, P.: Sensitivity of wavepackets to nonlinear effects: the role of the critical layer. J. Fluid Mech. 811, 95-137 (2017).

[16] Juvé, D., Sunyach, M., Comte-Bellot, G.: Intermittency of the noise emission in subsonic cold jets. J. Sound Vibr. 71, 319-32 (1980)

[17] Guj, G., Carley, M., Camussi, R.: Acoustic identification of coherent structures in a turbulent jet. J. Sound Vibr. 259, 1037-1065 (2003)

[18] Hileman JI, Thurow BS, Caraballo EJ, Samimy M. Large-scale structure evolution and sound emission in high-speed jets: real-time visualization with simultaneous acoustic measurements. J. Fluid Mech. 544, 277-307 (2005)

[19] Bogey, C., Bailly,C.: An analysis of the correlations between the turbulent flow and the sound pressure fields of subsonic jets. J. Fluid Mech. 583, 71-97 (2007)

[20] Kastner, J., Samimy, M., Hileman, J., Freund, J.B.: Comparison of Noise Mechanisms in High and Low Reynolds Number High-Speed Jets. AIAA J. 44, 2251-2258 (2006)

[21] Suponitsky, V., Sandham, N.D., Morfey, C.L.: Linear and nonlinear mechanisms of sound radiation by instability waves in subsonic jets. J. Fluid Mech. 658, 509-538 (2010)

[22] Bogey, C., Bailly, C. \& Juvé, D., 2003, Noise investigation of a high subsonic, moderate Reynolds number jet using a compressible LES, Theoretical and Computational Fluid Dynamics, 16(4), 273-297.

[23] D. G. Crighton and M. Gaster. Stability of slowly diverging jet flow. Journal of Fluid Mechanics, 77:397-413, 1976.

[24] Schmidt, O. T., Towne, A., Rigas, G., Colonius, T., Brès, G. A.: Spectral analysis of jet turbulence. J. Fluid Mech.855, 953-982 (2018)

[25] Cavalieri, A.V.G., Jordan, P., Agarwal, A., Gervais, Y.: Jittering wave-packet models for subsonic jet noise. J. Sound Vibr. 330, 4474-4492 (2011).

[26] A. V. G. Cavalieri, P. Jordan, and L. Lesshafft. Wave-packet models for jet dynamics and sound radiation. Applied Mechanics Reviews, 71(020802), 2019.

[27] A. V. G. Cavalieri and A. Agarwal. Coherence decay and its impact on sound radiation by wavepackets. Journal of Fluid Mechanics, 748:399-415, 2014.

[28] Y. B. Baqui, A. Agarwal, A. V. G. Cavalieri, and S. Sinayoko. A coherence-matched linear mechanism for subsonic jets. Journal of Fluid Mechanics, 776(235-267), 2015. 
[29] I. A. Maia, P. Jordan, A. V. G. Cavalieri, and V. Jaunet. Two-point wavepacket modelling of jet noise. Proceedings of the Royal Society A, 475(20190199), 2019.

[30] V. Jaunet, P. Jordan, and A. V. G. Cavalieri. Two-point coherence of wavepackets in turbulent jets. Physical Review Fluids, 2(024604), 2017.

[31] D. Breakey P. Jordan, A. V. G. Cavalieri, P. Nogueira, O. Léon, T. Colonius, and D. Rodríguez. Experimental study of turbulent-jet wave packets and their acoustic efficiency. Phys. Rev. Fluids, 2:124601, 2017.

[32] Kopiev V., Chernyshev S. "Simulation of azimuthal characteristics of turbulent jet noise by correlation model of quadrupole noise sources." International Journal of Aeroacoustics, Vol.13, No 1, 2014.

[33] Kopiev V., Faranosov, G. "On defining the jet noise source quadrupole structure on the basis of multiarray acoustic data and correlation theory", AIAA paper AIAA 2016-2806, 22nd AIAA/CEAS Aeroacoustics Conference, May 30 - June 1 2016, Lyon, France.

[34] Kearney-Fischer, M., Sinha, A., Samimy, M.: Intermittent Nature of Subsonic Jet Noise. AIAA J. 51, 1142-55 (2013)

[35] Kearney-Fischer, M.: A model function for jet noise events at aft angles and what it says about the statistical relationships of the events. J. Sound Vibr. 338, 217-236 (2015)

[36] Camussi, R., Di Marco, A., Castelain, T.: Statistical analysis of the hydrodynamic pressure in the near field of compressible jets. Int. J. Heat Fl. Flow 64, 1-9 (2017a)

[37] Camussi, R., Mancinelli, M., Di Marco, A.: Intermittency and stochastic modeling of hydrodynamic pressure fluctuations in the near field of compressible jets, Int. J. Heat Fl. Flow 68, 180-188 (2017b)

[38] Bogey, C.: Two-dimensional features of correlations in the flow and near pressure fields of Mach number 0.9 jets. AIAA Paper 2019-0806 (2019)

[39] Bogey, C., Marsden, O., and Bailly, C.: Large-Eddy Simulation of the flow and acoustic fields of a Reynolds number $10 \mathrm{e} 5$ subsonic jet with tripped exit boundary layers. Phys. Fluids 23, 035104 (2011)

[40] Bogey, C.: Grid sensitivity of flow field and noise of high-Reynolds-number jets computed by large-eddy simulation. Int. J. Aeroacoust. 17, 399-424 (2018)

[41] Bogey, C., and Sabatini, R.: Effects of nozzle-exit boundary-layer profile on the initial shearlayer instability, flow field and noise of subsonic jets. J. Fluid Mech. 876, 288-325 (2019) 
[42] Michalke, A., and Fuchs, H. V., "On turbulence and noise of an axisymmetric shear flow," J. Fluid Mech. , Vol. 70, No. 1, 1975, pp. 179-205.

[43] Arndt, R. E. A., Long, D. F., and Glauser, M. N., "The proper orthogonal decomposition of pressure fluctuations surrounding a turbulent jet," J. Fluid Mech. , Vol. 340, 1997, pp. 133.

[44] Jung, D., Gamard, S., and George, W. K., "Downstream evolution of the most energetic modes in a turbulent axisymmetric jet at high Reynolds number. Part 1 . The near-field region," J. Fluid Mech. , Vol. 514, 2004, pp. 173-204.

[45] Juve, D., Sunyach, M., and Comte-Bellot, G., "Filtered azimuthal correlations in the acoustic far field of a subsonic jet," AIAA J. , Vol. 17, No. 1, 1979, pp. 112-113. doi:https://doi.org/10.2514/3.61076.

[46] Farge, M.: Wavelet Transforms and their Applications to Turbulence. Ann. Rev. Fluid Mech. 24 395-458 (1992)

[47] Torrence, C., Compo, G.P.: A practical guide to wavelet analysis. Bull Am. Met. Soc. 79, 6178 (1998)

[48] Auger, F., Flandrin, P., Goncalves, P., Lemoine, O., Time-Frequency Toolbox Tutorial (2005)

[49] Mancinelli, M., Pagliaroli, T., Di Marco, A., Camussi, R., Castelain, T.: Wavelet decomposition of hydrodynamic and acoustic pressures in the near-field of the jet. J. Fluid Mech. 813, 716-749 (2017)

[50] Camussi, R., Guj, G.: Orthonormal wavelet decomposition of turbulent flows: intermittency and coherent structures. J. Fluid Mech. 348, 177-199 (1997)

[51] Meneveau, C.: Analysis of turbulence in the orthonormal wavelet representation. J. Fluid Mech. 232, 469-520 (1991)

[52] Grizzi, S., Camussi, R.: Wavelet analysis of near-field pressure fluctuations generated by a subsonic jet. J. Fluid Mech. 698, 93-124 (2012)

[53] Suzuki, T. M., Colonius, T.: Instability waves in a subsonic round jet detected using a nearfield phased microphone array. J. Fluid Mech. 565, 197-226 (2007)

[54] Me'tais O., Lesieur, M.: Spectral large-eddy simulations of isotropic and stably-stratified turbulence. J. Fluid Mech. 239, 157-194 (1992)

[55] Holzer M., Siggia, E.: Skewed, exponential pressure distributions from Gaussian velocities. Phys. Fluids A, 5:2525 (1993) 
[56] N. Cao, S. Chen, and G. D. Doolen. Statistics and structures of pressure in isotropic turbulence. Phys. Fluids, 11:2235-2250, 1999

[57] Feller, E., An introduction to probability theory and its applications. Wiley (1971)

[58] E. Mollo-Christensen. Measurements of near field pressure of subsonic jets. Technical report, DTCI, 1963.

[59] E. Mollo-Christensen. Jet noise and shear flow instability seen from an experimenter's viewpoint. Journal of Applied Mechanics, 34:1-7, 1967.

[60] Bogey, C. \& Pineau, P., 2019, Potential-core closing of temporally developing jets at Mach numbers between 0.3 and 2: Scaling and conditional averaging of flow and sound fields, Phys. Rev. Fluids, , 124601, 1-25.

[61] Pineau, P. \& Bogey, C., 2019, Steepened Mach waves near supersonic jets: study of azimuthal structure and generation process using conditional averages, J. Fluid Mech., 880, 594-619.

[62] Bogey, C., 2019, On noise generation in low Reynolds number temporal round jets at a Mach number of 0.9, J. Fluid Mech., 859, 1022-1056. 